\title{
Similarity of energy structure functions in decaying homogeneous isotropic turbulence
}

\author{
By R. A. ANTONIA ${ }^{1}$, R. J. SMALLEY ${ }^{2}$, T. ZHOU ${ }^{3}$, \\ F. ANSELMET AND L. DANAILA \\ ${ }^{1}$ Discipline of Mechanical Engineering, University of Newcastle, NSW 2308, Australia \\ ${ }^{2}$ Energy and Resources Research Institute, University of Leeds, Leeds, LS2 9JT, UK \\ ${ }^{3}$ Department of Mechanical \& Production Engineering, Nanyang \\ Technological University, Singapore 639798 \\ ${ }^{4}$ IRPHE, Université d'Aix-Marseille I \& II, 13384 Marseille, France \\ ${ }^{5}$ CORIA, Avenue de l'Université, BP12, 76801, Saint Etienne du Rouvray, France
}

(Received 4 March 2002 and in revised form 28 February 2003)

An equilibrium similarity analysis is applied to the transport equation for $\left\langle(\delta q)^{2}\right\rangle$ $\left(\equiv\left\langle(\delta u)^{2}\right\rangle+\left\langle(\delta v)^{2}\right\rangle+\left\langle(\delta w)^{2}\right\rangle\right)$, the turbulent energy structure function, for decaying homogeneous isotropic turbulence. A possible solution requires that the mean energy $\left\langle q^{2}\right\rangle$ decays with a power-law behaviour $\left(\left\langle q^{2}\right\rangle \sim x^{m}\right)$, and the characteristic length scale, which is readily identifiable with the Taylor microscale, varies as $x^{1 / 2}$. This solution is identical to that obtained by George (1992) from the spectral energy equation. The solution does not depend on the actual magnitude of the Taylormicroscale Reynolds number $R_{\lambda}\left(\sim\left\langle q^{2}\right\rangle^{1 / 2} \lambda / v\right) ; R_{\lambda}$ should decay as $x^{(m+1) / 2}$ when $m<-1$. The solution is tested at relatively low $R_{\lambda}$ against grid turbulence data for which $m \simeq-1.25$ and $R_{\lambda}$ decays as $x^{-0.125}$. Although homogeneity and isotropy are poorly approximated in this flow, the measurements of $\left\langle(\delta q)^{2}\right\rangle$ and, to a lesser extent, $\left\langle(\delta u)(\delta q)^{2}\right\rangle$, satisfy similarity reasonably over a significant range of $r / \lambda$, where $r$ is the streamwise separation across which velocity increments are estimated. For this range, a similarity-based calculation of the third-order structure function $\left\langle(\delta u)(\delta q)^{2}\right\rangle$ is in reasonable agreement with measurements. Kolmogorov-normalized distributions of $\left\langle(\delta q)^{2}\right\rangle$ and $\left\langle(\delta u)(\delta q)^{2}\right\rangle$ collapse only at small $r$. Assuming homogeneity, isotropy and a Batchelor-type parameterization for $\left\langle(\delta q)^{2}\right\rangle$, it is found that $R_{\lambda}$ may need to be as large as $10^{6}$ before a two-decade inertial range is observed.

\section{Introduction}

The problem of determining how energy decays in homogeneous isotropic turbulence has been around for some time now. Taylor (1935) was first to describe the concept of isotropy and apply it to the streamwise decay of energy in grid turbulence. His work was extended by von Kármán \& Howarth (1938) who started from the equations of motion to derive a relation, in the form of a partial differential equation, between the double $\left(\equiv B_{u, u}=\langle u(x+r) u(x)\rangle\right)$ and triple $\left(\equiv B_{u u, u}=\left\langle u^{2}(x+r) u(x)\right\rangle\right)$ velocity correlation functions:

$$
\frac{\partial B_{u, u}}{\partial t}+2\left(\frac{\partial B_{u u, u}}{\partial r}+\frac{4 B_{u u, u}}{r}\right)=2 v\left(\frac{\partial^{2} B_{u, u}}{\partial r^{2}}+\frac{4}{r} \frac{\partial B_{u, u}}{\partial r}\right) .
$$


These authors also investigated the solution of this equation when $B_{u u, u}(r)$ is neglected and $B_{u, u}(r)$ is self-preserving, i.e. its shape remains similar during decay, the only change occurring through its scale. The idea of self-preservation was subsequently adopted by a number of authors (e.g. Dryden 1943; Batchelor 1948; Batchelor \& Townsend 1947, 1948; Lin 1948; von Kármán \& Lin 1949; Goldstein 1951; Stewart \& Townsend 1951; see also Monin \& Yaglom 1975 and Hinze 1975) for investigating the initial and final periods of decay, in the context of either velocity correlation functions or energy spectra. The most detailed listing of different types of selfpreserving energy decay scenarios was provided by Batchelor (1948) for correlation functions and Panchev (1971) for energy spectra. In the final period of decay, when the Reynolds number is quite small, Batchelor found, by linearizing the equation, that $\left\langle q^{2}\right\rangle \sim\left(t-t_{0}\right)^{-5 / 2}$ where $t_{0}$ is the temporal origin for the power-law decay; $\left\langle q^{2}\right\rangle$, twice the turbulent kinetic energy, is defined by

$$
\left\langle q^{2}\right\rangle=2 \int_{0}^{\infty} E(k) \mathrm{d} k .
$$

In (1.2), $E(k)$ is the three-dimensional energy spectrum and $k$ is the magnitude of the wavenumber vector $\boldsymbol{k}$. George (1992) showed that $\left\langle q^{2}\right\rangle$ decayed as $t^{-5 / 2}$ as $k \rightarrow 0$ provided the Loitsianskii invariant was satisfied or, equivalently, that $E(k) \sim k^{4}$. In the initial period and for infinite Reynolds numbers, Kolmogorov (1941a) found that $\left\langle q^{2}\right\rangle \sim\left(t-t_{0}\right)^{-10 / 7}$. A more complete solution $\left(\left\langle q^{2}\right\rangle \sim\left(t-t_{0}\right)^{-1}\right)$, in which viscosity is retained in the von Kármán-Howarth equation, was found by Dryden (1943), the Taylor microscale $\lambda$ (defined in §2) being the relevant length scale; like von Kármán and Howarth, Dryden required $R_{\lambda}$ to be constant, which in turn requires a ' -1 ' decay since $\lambda \sim\left(t-t_{0}\right)^{1 / 2}$. As noted by Speziale \& Bernard (1992), this solution was also implicit in Batchelor's (1948) analysis but dismissed because Loitsianskii's integral was not invariant (Proudman \& Reid 1954). Saffman (1967) showed that the integral $\int_{0}^{\infty} r^{2} B_{u, u}(r) \mathrm{d} r$ was invariant, and used this as well as an additional assumption about the form of $E(k)$ to predict $\left\langle q^{2}\right\rangle \sim\left(t-t_{0}\right)^{-6 / 5}$ and $\left\langle q^{2}\right\rangle \sim\left(t-t_{0}\right)^{-3 / 2}$ for the initial and final periods of decay respectively. The $t^{-3 / 2}$ result was obtained in George (1992) without a model for $E(k)$. Tatsumi (1980) and Mohamed \& LaRue (1990), who reviewed a significant amount of available data for decaying turbulence downstream of a grid, found that, for the initial period, the majority of these exhibited a $\left\langle u^{2}\right\rangle \sim\left(x-x_{0}\right)^{m}$ behaviour, with $m \simeq-1.3$ rather than $m=-1$. This latter exponent was initially thought to be correct (an interesting discussion of this is given in George et al. 2001) but, later, consensus favoured $m<-1$ due mainly to the measurements of Corrsin (1963) (see George et al.) and Comte-Bellot \& Corrsin (1966, 1971). Direct numerical simulations (DNS) have yielded a much larger range of values for $m$, typically between -1 and -2.5 (George et al. 2001), apparently reflecting, at least in part, the greater variation in initial conditions than that which occurs in experiments. While decaying box turbulence simulations can potentially approximate homogeneous isotropic turbulence more closely than grid turbulence, the finite size of the DNS box can seriously affect the lowest wavenumbers of the energy spectrum. This can in turn affect $\left\langle q^{2}\right\rangle$ (and therefore the exponent $m$ ) and, to a greater extent, the integral length scale $L$ (George et al. 2001; Wang \& George 2002). In grid experiments, one cannot discount the effect the size of a tunnel working section may have on the rate of growth of $L$. A more general discussion of the effect of finite boundaries is given in George (1999).

George $(1987,1992)$ - the latter paper will hereafter be referred to as G92 re-examined the self-preserving decay of homogeneous isotropic turbulence by 
considering the spectral energy equation

$$
\frac{\partial E(k)}{\partial t}=T(k)-2 v k^{2} E(k),
$$

where $T(k)$ is the nonlinear spectral transfer function. He obtained the solution $\left\langle q^{2}\right\rangle \sim\left(t-t_{0}\right)^{m}$, independently of the magnitude of the Reynolds number, for $m \leqslant-1$. Speziale \& Bernard (1992) carried out a fixed point analysis on an equation which combines the von Kármán-Howarth equation with the transport equation for $\langle\epsilon\rangle$, the mean energy dissipation rate. They found that $\left\langle q^{2}\right\rangle \sim\left(t-t_{0}\right)^{-1}$ is the asymptotic high-Reynolds-number solution whilst $\left\langle q^{2}\right\rangle \sim\left(t-t_{0}\right)^{m}$ is achieved as $t \rightarrow \infty$ and the Reynolds number approaches 0 . It should be made clear however that these authors (also Zhou \& Speziale 1998, who considered the equation for the two-point double velocity correlation) assumed in essence that the velocity derivative skewness $S$ $\left(\equiv-\left\langle(\partial u / \partial t)^{3}\right\rangle /\left\langle(\partial u / \partial t)^{2}\right\rangle^{3 / 2}\right)$ remains constant with $t$ and deduced that $R_{\lambda}$ is constant and $\left\langle q^{2}\right\rangle \sim\left(t-t_{0}\right)^{-1}$. This follows immediately from G92, which says that only the product $S R_{\lambda}$ is constant with no further assumptions about either $S$ or $R_{\lambda}$.

In the specific context of grid turbulence for which $m$ is typically smaller than -1 and the Reynolds number is generally small (this is usually the case for passive grids), the G92 solution is attractive. When $m=-1$, G92 is consistent with asymptotic solutions (Kolmogorov 1941a; von Kármán \& Howarth 1938; Speziale \& Bernard 1992). In support of the $m<-1$ similarity solution, George examined both the one-dimensional spectra of $u$ measured by Comte-Bellot \& Corrsin (1971) and threedimensional energy spectra which were calculated from the measured $u$ spectra via isotropy. This calculation should be approximately valid in the context of the ComteBellot \& Corrsin experiment where a secondary contraction was placed downstream of the grid and isotropy was satisfied approximately at both large and small scales. Although reasonable collapse of the spectra was found using $\left\langle q^{2}\right\rangle^{1 / 2}$ and $\lambda$, it was not a significant improvement over that based on the Kolmogorov length $\eta \equiv v^{3 / 4}\langle\epsilon\rangle^{-1 / 4}$ and velocity $U_{K} \equiv v^{1 / 4}\langle\epsilon\rangle^{1 / 4}$ scales (published spectra, obtained over a wide range of flows, support this scaling at sufficiently large wavenumbers, e.g. Saddoughi \& Veeravalli 1994 ) or that which uses the integral length scale $L$ (defined from $E(k),(4.1)$ in $\S 4$ ) and $\left\langle q^{2}\right\rangle^{1 / 2}$. Equally good collapse of the spectra with any of the three previously mentioned sets of scales would be expected if $R_{\lambda}\left(\sim\left\langle q^{2}\right\rangle^{1 / 2} \lambda / \nu\right.$; a more complete definition is given in §2) remains constant during decay since $\lambda / \eta \sim R_{\lambda}^{1 / 2}$ (Tennekes \& Lumley 1972). Similarly, if a pseudo-integral length scale $L_{\epsilon} \equiv C_{\epsilon}\left\langle q^{2} / 3\right\rangle^{3 / 2} /\langle\epsilon\rangle$ is used (e.g. Batchelor 1953; Tennekes \& Lumley 1972; the constant $C_{\epsilon}$ depends on the Reynolds number and, more generally, on initial conditions; the relevance of $L_{\epsilon}$ to the present experiment will be discussed in $\S 4$ ), then $L_{\epsilon} / \lambda \sim R_{\lambda}$ and $L$ is proportional to $\lambda$ only if $R_{\lambda}$ remains constant. Constancy of $R_{\lambda}$ would require that $m=-1$ since $\left\langle q^{2}\right\rangle^{1 / 2} \sim\left(x-x_{0}\right)^{m / 2}$ and $\lambda \sim\left(x-x_{0}\right)^{1 / 2}$. It should also be noted that $\left\langle q^{2}\right\rangle^{1 / 2} \sim U_{K} R_{\lambda}^{1 / 2}$, so that $\left\langle q^{2}\right\rangle^{1 / 2}$ is proportional to $U_{K}$ if $R_{\lambda}$ is constant; similarity of the von Kármán-Howarth equation using $U_{K}$ and $\eta$ was shown to be possible by Lin (1948) when $U_{K}^{2} \sim\left(t-t_{0}\right)$ and $\eta \sim\left(t-t_{0}\right)^{1 / 2}$.

The G92 analysis differs from the earlier work (Dryden 1943; Batchelor 1948) only through the treatment of $B_{u u, u}$ in (1.1) or, equivalently, $T(k)$ in (1.2). G92 argued that it was more rigorous not to specify the same scale for $B_{u u, u}$ as for $B_{u, u}$ (or $E(k)$ ); the analysis yielded $R_{\lambda}^{-1}\left\langle q^{2}\right\rangle^{3 / 2}$ (instead of $\left\langle q^{2}\right\rangle^{3 / 2}$ ) as the 'scale' for $B_{u u, u}$ (or $T(k)$ ). Batchelor \& Townsend (1948) calculated $B_{u u, u}$, using (1.1) and a selfpreserving form of $B_{u, u}$. This calculation was however in quite poor agreement with 
the measured distribution. Corrsin (1963) presented a calculation of $B_{u u, u}$, apparently based on $\left\langle q^{2}\right\rangle-\lambda$ similarity and the distribution of $B_{u, u}$, as measured by Stewart (1951). The agreement between calculation and measurements was better than that reported by Batchelor \& Townsend (1948). Nevertheless, the calculation deviated significantly from measurements, both at small and, more particularly, large $r / \lambda$. At small $r / \lambda$, the calculated distribution increased more rapidly than the measured one, the former peaking at $r / \lambda \simeq 1.5$ and the latter at $r / \lambda \simeq 2.5$. Corrsin attributed the lack of agreement for $r / \lambda \gtrsim 2$ to a departure from isotropy of the large scales. It should also be mentioned that Stewart's measurements of $B_{u, u}$ did not satisfy $\left\langle q^{2}\right\rangle-\lambda$ similarity, while the measured (normalized) distributions of $B_{u u, u}$, at different $x$, exhibited significant variability. Stewart (see also Stewart \& Townsend 1951) noted the severe difficulties encountered in the measurement of triple correlations. Monin \& Yaglom (1975) examined the Stewart \& Townsend (1951) data in the context of self-preservation of the structure functions, as originally carried out by Lin (1948). An obvious advantage of this approach is that the second-order structure function must go to zero at small $r$ and to a constant at large $r$ (the normalizing velocity scale can be chosen to ensure that this constant is independent of $x$ ). They found that the data for $\left\langle(\delta u)^{2}\right\rangle$ could be made to satisfy self-preservation closely after suitable normalizing velocity scales were chosen; the distributions of $\left\langle(\delta u)^{3}\right\rangle$ also collapsed reasonably well for $r / \lambda \lesssim 4$. As in Monin \& Yaglom, we focus on structure functions, although their empirical approach is avoided here. Instead, we follow a route analogous to G92 and consider the equation which corresponds to (1.3) in physical space. From an experimental viewpoint, this has the advantage of allowing possible similarity solutions to be tested more readily than in the spectral domain since measurements of $T(k)$ and $E(k)$ are now avoided. Similarity solutions to (1.3) can be tested using DNS data (e.g. George et al. 2001). It can also be argued that structure functions are more sensitive than spectra for testing different types of similarity solutions. Whereas integral constraints apply to spectra, e.g. (1.2), the constraint on structure functions is local.

The equation for velocity structure functions, which corresponds fully to (1.1) and (1.3), is (Danaila et al. 1999)

$$
-\left\langle(\delta u)^{3}\right\rangle+6 v \frac{\mathrm{d}}{\mathrm{d} r}\left\langle(\delta u)^{2}\right\rangle-3 \frac{U}{r^{4}} \int_{0}^{r} s^{4} \frac{\partial}{\partial x}\left\langle(\delta u)^{2}\right\rangle \mathrm{d} s=\frac{4}{5}\langle\epsilon\rangle r,
$$

where $\delta u \equiv u(x+r)-u(x)$ is the difference in streamwise velocity fluctuation between two points in space separated by $r$ along the streamwise direction, $U$ is the mean streamwise velocity, $\langle\epsilon\rangle$ is the mean kinetic energy dissipation rate and $s$ is a dummy separation variable. It was shown that grid turbulence data satisfied (1.4) reasonably well, after a self-preserving approximation and the assumption of global isotropy was made to estimate $\partial\left\langle(\delta u)^{2}\right\rangle / \partial x$. An equation for $\left\langle(\delta q)^{2}\right\rangle$ was derived in Danaila, Anselmet \& Antonia (2002):

$$
-\left\langle(\delta u)(\delta q)^{2}\right\rangle+2 v \frac{\mathrm{d}}{\mathrm{d} r}\left\langle(\delta q)^{2}\right\rangle-\frac{U}{r^{2}} \int_{0}^{r} s^{2} \frac{\partial}{\partial x}\left\langle(\delta q)^{2}\right\rangle \mathrm{d} s=\frac{4}{3}\langle\epsilon\rangle r .
$$

Note that we have preferred to write (1.4) and (1.5) in terms of $x$ rather than time mainly to facilitate the comparison between analytical results and grid turbulence measurements. While (1.4) represents the equivalent expression in physical space to (1.3), (1.5) requires a less restrictive isotropy hypothesis than (1.4). In addition, as was emphasized in Danaila et al. (2002), this latter equation reduces for very large scales to the homogeneous definition of $\langle\epsilon\rangle$, i.e. $\langle\epsilon\rangle_{\text {hom }} \equiv 3 \nu\left\langle(\partial q / \partial x)^{2}\right\rangle$. 
In $\S 2$, we consider the conditions for which (1.5) satisfies equilibrium similarity (the terminology of G92 is retained here). We then test this solution using measurements downstream of a grid. The availability of $\left\langle q^{2}\right\rangle$ and $\langle\epsilon\rangle$ in this experiment allow more general definitions of $\lambda$ and $R_{\lambda}$ than would be possible with only $\left\langle u^{2}\right\rangle$ and $\langle\epsilon\rangle_{\text {iso }}$, the isotropic value of $\langle\epsilon\rangle$. The basic characteristics of the flow are discussed in $\S 4$. Section 5 tests the similarity forms of both $\left\langle(\delta q)^{2}\right\rangle$ and $\left\langle(\delta u)(\delta q)^{2}\right\rangle$. Comparison is also made between the measured distribution of $\left\langle(\delta u)(\delta q)^{2}\right\rangle$ and that calculated using (1.5), via measurements of $\left\langle(\delta q)^{2}\right\rangle$ and $\langle\epsilon\rangle$. In $\S 6$, the effect of Reynolds number on the calculation of $\left\langle(\delta u)(\delta q)^{2}\right\rangle$ is tested by assuming a parameterization for $\left\langle(\delta q)^{2}\right\rangle$ for homogeneous isotropic turbulence.

It should be made clear from the outset that the present equilibrium similarity solution $(\$ 2)$, like that of G92, does not require the Reynolds number to be large. Although it would be desirable to test the theory against data in which there is a large separation of scales between $L$ and $\eta$, the theory is valid for any value of the Reynolds number and does not constrain $R_{\lambda}$ to be constant (with respect to time or distance) for a specified set of initial conditions. We also emphasize that whilst G92 focused on the spectral domain, our approach deals with physical space and is more readily amenable to testing against laboratory measurements.

\section{Similarity solution of the energy structure function equation}

We first examine the conditions under which (1.5) can satisfy similarity. Following G92,

$$
\left\langle(\delta q)^{2}\right\rangle=V f\left(\frac{r}{\mathscr{L}}\right)
$$

and

$$
-\left\langle(\delta u)(\delta q)^{2}\right\rangle=H g\left(\frac{r}{\mathscr{L}}\right),
$$

where $\mathscr{L}$ is a characteristic length scale, $V$ (with dimensions of velocity squared) and $H$ (with dimensions of velocity cubed) are scales that characterize $\left\langle(\delta q)^{2}\right\rangle$ and $\left\langle(\delta u)(\delta q)^{2}\right\rangle ; \mathscr{L}, V$ and $H$ depend only on $x$. The dimensionless functions $f$ and $g$ depend not only on $r / \mathscr{L}$ but also on initial conditions (for simplicity of expression, this latter dependence is not indicated). After substituting (2.1) and (2.2) into (1.5), we obtain

$$
H g+2 \frac{\nu V}{\mathscr{L}} f^{\prime}+\frac{U \mathscr{L}^{2}}{r^{2}} V \frac{\mathrm{d} \mathscr{L}}{\mathrm{d} x} \Gamma_{1}-\frac{U \mathscr{L}^{3}}{r^{2}} \frac{\mathrm{d} V}{\mathrm{~d} x} \Gamma_{2}=-\frac{2}{3} U \mathscr{L} \frac{\mathrm{d}\left\langle q^{2}\right\rangle}{\mathrm{d} x} \frac{r}{\mathscr{L}},
$$

where a prime denotes differentiation with respect to $r / \mathscr{L}$ and $\Gamma_{1}$ and $\Gamma_{2}$ are given by

$$
\begin{aligned}
\Gamma_{1} & \equiv \int_{0}^{r / \mathscr{L}}\left(\frac{s}{\mathscr{L}}\right)^{3} f^{\prime} d\left(\frac{s}{\mathscr{L}}\right), \\
\Gamma_{2} & \equiv \int_{0}^{r / \mathscr{L}}\left(\frac{s}{\mathscr{L}}\right)^{2} f d\left(\frac{s}{\mathscr{L}}\right) .
\end{aligned}
$$

In (2.3), $\langle\epsilon\rangle$ was replaced by its corresponding value for decaying isotropic turbulence:

$$
\langle\epsilon\rangle=-\frac{U}{2} \frac{\mathrm{d}\left\langle q^{2}\right\rangle}{\mathrm{d} x}
$$


and the dependence of both $f$ and $g$ on $\tilde{r} \equiv r / \mathscr{L}$ is implicitly assumed. After multiplication by $(\mathscr{L} / v V),(2.3)$ becomes

$$
\left[\frac{H \mathscr{L}}{v V}\right] g+[2] f^{\prime}+\left[\frac{U \mathscr{L}}{v} \frac{\mathrm{d} \mathscr{L}}{\mathrm{d} x}\right] \tilde{r}^{-2} \Gamma_{1}-\left[\frac{U \mathscr{L}^{2}}{v V} \frac{\mathrm{d} V}{\mathrm{~d} x}\right] \tilde{r}^{-2} \Gamma_{2}=-\frac{2}{3}\left[\frac{U \mathscr{L}^{2}}{v V} \frac{\mathrm{d}\left\langle q^{2}\right\rangle}{\mathrm{d} x}\right] \tilde{r} .
$$

For equilibrium similarity (e.g. G92) all the terms within square brackets must evolve in the streamwise direction in exactly the same way. Since the second term of these is constant, all the other tems must also be constant:

$$
\begin{aligned}
& \frac{H \mathscr{L}}{v V}=\text { constant, } \\
& \frac{U \mathscr{L}}{v} \frac{\mathrm{d} \mathscr{L}}{\mathrm{d} x}=a, \\
& \frac{U \mathscr{L}^{2}}{v V} \frac{\mathrm{d} V}{\mathrm{~d} x}=b,
\end{aligned}
$$

and

$$
\frac{U \mathscr{L}^{2}}{v V} \frac{\mathrm{d}\left\langle q^{2}\right\rangle}{\mathrm{d} x}=\text { constant }
$$

Since $U$ is constant, integration of (2.9) immediately yields

$$
\mathscr{L}^{2}-\mathscr{L}_{0}^{2}=\frac{2 a v\left(x-x_{0}\right)}{U}
$$

with $\mathscr{L}_{0}=0$ at $x=x_{0}$. By comparing (2.10) and (2.11), then

$$
V \sim\left\langle q^{2}\right\rangle .
$$

Conditions (2.10) and (2.12) yield, after integration with respect to $x$,

$$
\frac{V}{V_{i}}=\left(\frac{x-x_{0}}{x_{i}-x_{0}}\right)^{b / 2 a}
$$

where $V=V_{i}$ at any point $x=x_{i}$, except $x_{i}=x_{0}$. Relations (2.13) and (2.14) indicate that a possible similarity solution of (1.5) is

$$
\left\langle q^{2}\right\rangle=A\left(x-x_{0}\right)^{m},
$$

with $m=b / 2 a$. The Taylor microscale $\lambda$ is defined here as

$$
\lambda^{2}=5 v \frac{\left\langle q^{2}\right\rangle}{\langle\epsilon\rangle} .
$$

Using (2.6) and (2.15), $\lambda^{2}$ reduces to

$$
\lambda^{2}=-\frac{10 v}{m} \frac{\left(x-x_{0}\right)}{U} .
$$

Comparison of (2.12) and (2.17) suggests that the characteristic length scale $\mathscr{L}$ can be identified with $\lambda$. With $V \equiv\left\langle q^{2}\right\rangle$ and $\mathscr{L} \equiv \lambda$, (2.8) implies that

$$
H \sim v \frac{\left\langle q^{2}\right\rangle}{\lambda} \quad \text { or } \quad H \sim R_{\lambda}^{-1}\left\langle q^{2}\right\rangle^{3 / 2} .
$$



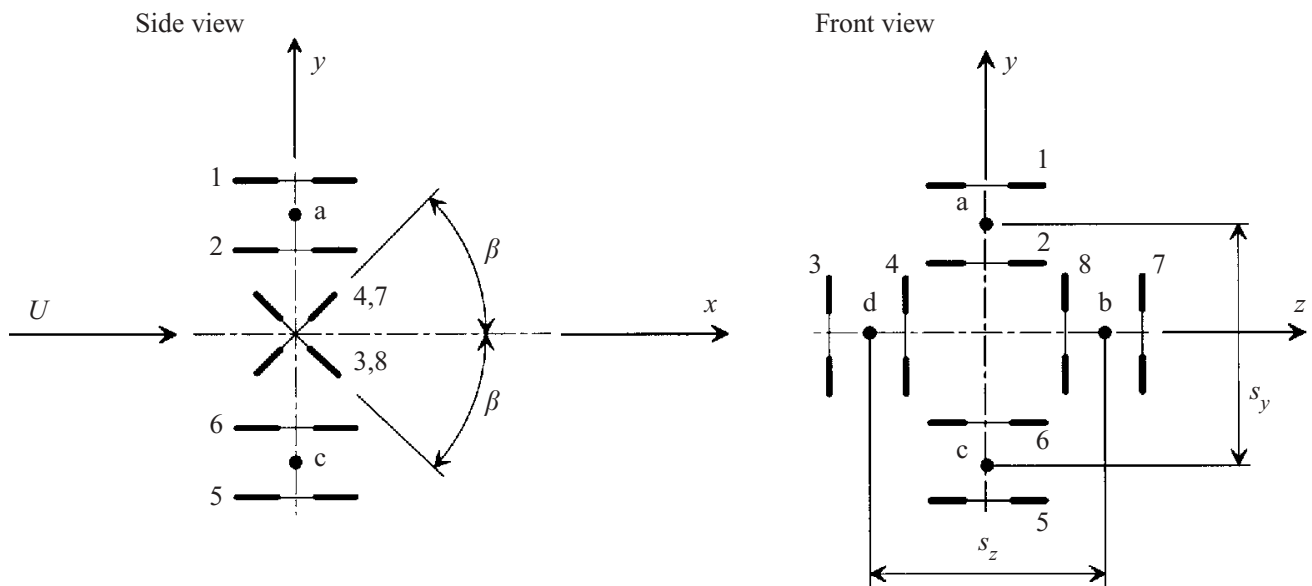

FIGURE 1. Schematic arrangement of the three-component hot-wire vorticity probe. The separation between wires in each of the four X-probes is about $0.6 \mathrm{~mm}$. All wires have a diameter of $2.5 \mu \mathrm{m}$ and a length of about $0.5 \mathrm{~mm}$. The separations $S_{y}$ and $S_{z}$ are approximately $2.1 \mathrm{~mm}$ and $2.2 \mathrm{~mm}$ respectively. The angle $\beta$ is nominally $45^{\circ}$. Points a, b, c, d designate the geometric centres of the four X-probes.

$R_{\lambda}$ is defined here by

$$
R_{\lambda}=\frac{\left\langle q^{2}\right\rangle^{1 / 2}}{3^{1 / 2}} \frac{\lambda}{v}
$$

With $V \equiv\left\langle q^{2}\right\rangle, H \equiv 3^{-1 / 2} R_{\lambda}^{-1}\left\langle q^{2}\right\rangle^{3 / 2}$ (the factor $3^{-1 / 2}$ is included to simplify the final expression for $g$ ) and $\mathscr{L} \equiv \lambda,(2.7)$ can, after some manipulation, be rewritten as

$$
g=\frac{20}{3} \tilde{r}+5 \frac{\tilde{r}^{-2}}{m} \Gamma_{1}-10 \tilde{r}^{-2} \Gamma_{2}-2 f^{\prime} .
$$

This indicates that $g$ can be uniquely determined once $f$, the normalized second-order structure function, and $m$, the power-law exponent describing the rate of decay of $\left\langle q^{2}\right\rangle$, are known. The dependence of $g$ on initial conditions occurs implicitly through $f$ and $m$, both of which depend on the initial conditions.

\section{Experimental details}

The present measurements were made with a three-component vorticity probe, consisting of an arrangement comprising four X-wires (a sketch of this probe is shown in figure 1; this probe was first used by Antonia, Zhou \& Zhu 1998). Two of these are in the $(x, y)$-plane, parallel to each other, and separated by a distance $S_{z}$ (in the $z$-direction) of about $2.2 \mathrm{~mm}$. The other two are in the $(x, z)$-plane, also parallel to each other, and separated by a distance $S_{y}$ (in the $y$-direction) of about $2.1 \mathrm{~mm}$. The wires were etched from Wollaston $(\mathrm{Pt}-10 \% \mathrm{Rh})$ material to a diameter of $2.5 \mu \mathrm{m}$ and a nominal length of $0.5 \mathrm{~mm}$. Each wire was inclined at an effective angle of about $45^{\circ}$ to the mean flow direction.

For the biplane grid, consisting of $4.76 \mathrm{~mm}$ diameter rods, the mesh size $M$ or centre-to-centre spacing of the rods was $24.76 \mathrm{~mm}$ and the solidity of the grid was about 0.35 . The grid was located immediately downstream of the contraction (area ratio of 9:1) of an open-circuit low-speed wind tunnel and at the entrance of a working 
section $(350 \mathrm{~mm} \times 350 \mathrm{~mm} \times 2.4 \mathrm{~m}$ in length). The probe was traversed along the centreline of the working section between $x / M=20$ and $x / M=80$ (here, $x$ is the longitudinal distance downstream of the grid). The longitudinal mean velocity $U$ was constant $\left(\simeq 12.08 \mathrm{~m} \mathrm{~s}^{-1}\right)$ throughout the working section. The Reynolds number $R_{M}$ was equal to 19935 . The Kolmogorov length scale $\eta$, estimated using (2.6), increased from about $0.17 \mathrm{~mm}$ at $x / M=20$ to $0.37 \mathrm{~mm}$ at $x / M=80$. The ratio, with respect to $\eta$, of the largest separation $(2.2 \mathrm{~mm})$ between a pair of X-wires varied between about $13(x / M=20)$ and $6(x / M=80)$. Consequently, the resolution for the measurements of the vorticity fluctuations was poor, especially at the smaller values of $x / M$, and measured spectra of $\omega_{1}, \omega_{2}$ and $\omega_{3}$ had to be corrected. The corrections were discussed in Antonia et al. (1998); after correction, the three vorticity variances provided an estimate of $\langle\epsilon\rangle$ denoted by $\left\langle\epsilon_{h}\right\rangle$ ( $\equiv \nu\left\langle\omega_{i}^{2}\right\rangle$ for homogeneous turbulence) that agreed within $10 \%$ with that obtained using (2.6). Statistics of vorticity fluctuations have been shown to be in satisfactory agreement with those estimated from decaying box turbulence DNS data (Antonia, Orlandi \& Zhou 2002a), notwithstanding the difference in initial conditions between simulation and experiment. Each individual pair of X-wires provided data for $u, v, w$ with adequate spatial resolution since the separation between the inclined wires in each X-probe was about $0.6 \mathrm{~mm}$. The majority of the results presented in this paper use velocity fluctuations determined from only one combination of two adjoining pairs of X-wires (second-order moments of velocity statistics are essentially unchanged for any of the four combinations of adjoining X-wires).

The hot wires were operated with in-house constant-temperature circuits at an overheat ratio of 0.5. Output signals from these circuits were passed through buckand-gain amplifiers and low-pass filtered at a cut-off frequency $f_{c}$; its magnitude decreased as $x / M$ increased ( $f_{c}$ was $10 \mathrm{kHz}$ at $x / M=20$ and $5 \mathrm{kHz}$ at $x / M=40$ ). The selection of $f_{c}$ was finalized once the frequency corresponding to the onset of electronic noise was identified (Antonia et al. 1998). The magnitude of $f_{c}$ was quite close to that of the Kolmogorov frequency $f_{K}(\equiv U / 2 \pi \eta)$. The filtered signals were sampled at a frequency $f_{s} \geqslant 2 f_{c}$ and digitized using a 12 bit A-D converter. The record duration was $65 \mathrm{~s}$.

Uncertainties in $U$ and r.m.s. velocity fluctuations were inferred from errors in the hot-wire calibration data and the scatter (20:1 odds) observed from several repetitions of particular experimental runs. The uncertainty in $U$ is about $\pm 2 \%$ while the uncertainties in $\left\langle u^{2}\right\rangle^{1 / 2},\left\langle v^{2}\right\rangle^{1 / 2}$ and $\left\langle w^{2}\right\rangle^{1 / 2}$ are approximately $\pm 4.5 \%, \pm 5 \%$ and $\pm 5 \%$ respectively. The resulting uncertainties in $\left\langle q^{2}\right\rangle$ and $\langle\epsilon\rangle$ are $\pm 7 \%$ and $\pm 14 \%$ respectively.

\section{Characteristics of grid turbulence}

The difficulty of estimating the decay exponent $m$ by fitting a limited number of data points (for $\left\langle q^{2}\right\rangle$ ) is well known. George et al. (2001) noted that this type of fitting is sensitive to parameter forcing as there are two parameters $\left(A\right.$ and $\left.x_{0}\right)$ which may be varied to achieve a desired value for $m$. They also indicated that only over a range where $\mathrm{d} \lambda^{2} / \mathrm{d} x$ is constant (with $\lambda$ defined by (2.16)) can a power-law exist and similarity conditions be tested. While this may be appropriate for DNS data with quite small time steps, it is more difficult to implement with grid turbulence data, usually taken only at a finite number of $x$ locations and relatively coarse $x$ steps. To avoid this difficulty, the data for $\lambda^{2} /\left(x-x_{0}\right)$ were plotted (figure 2) for different choices of $x_{0}$. Figure 2 indicates that the best plateau over the range 


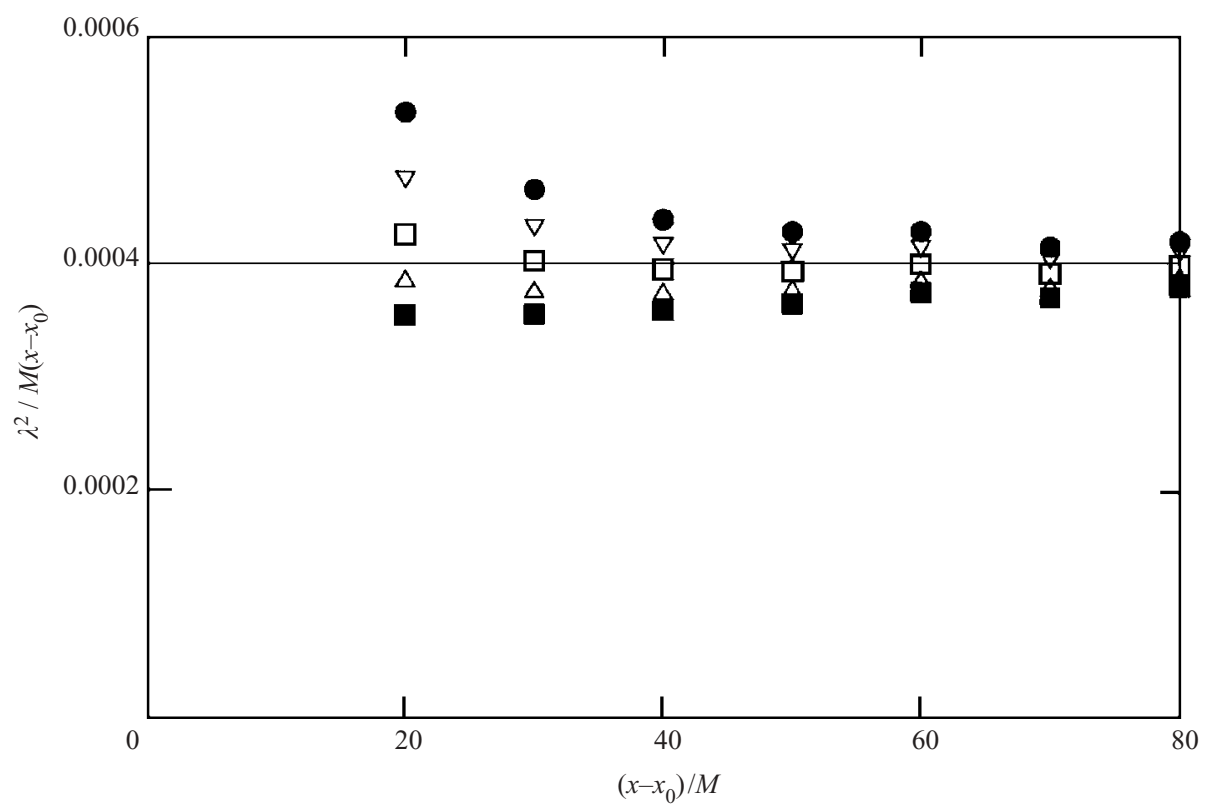

Figure 2. Ratio $(\lambda / M)^{2} /\left[\left(x-x_{0}\right) / M\right]$ for different choices of $x_{0}$. The horizontal line corresponds to (2.17) when $m=-1.25 . \bullet, x_{0} / M=4 ; \nabla, 2 ; \square, 0 ; \triangle,-2 ; \mathbf{\square},-4$.

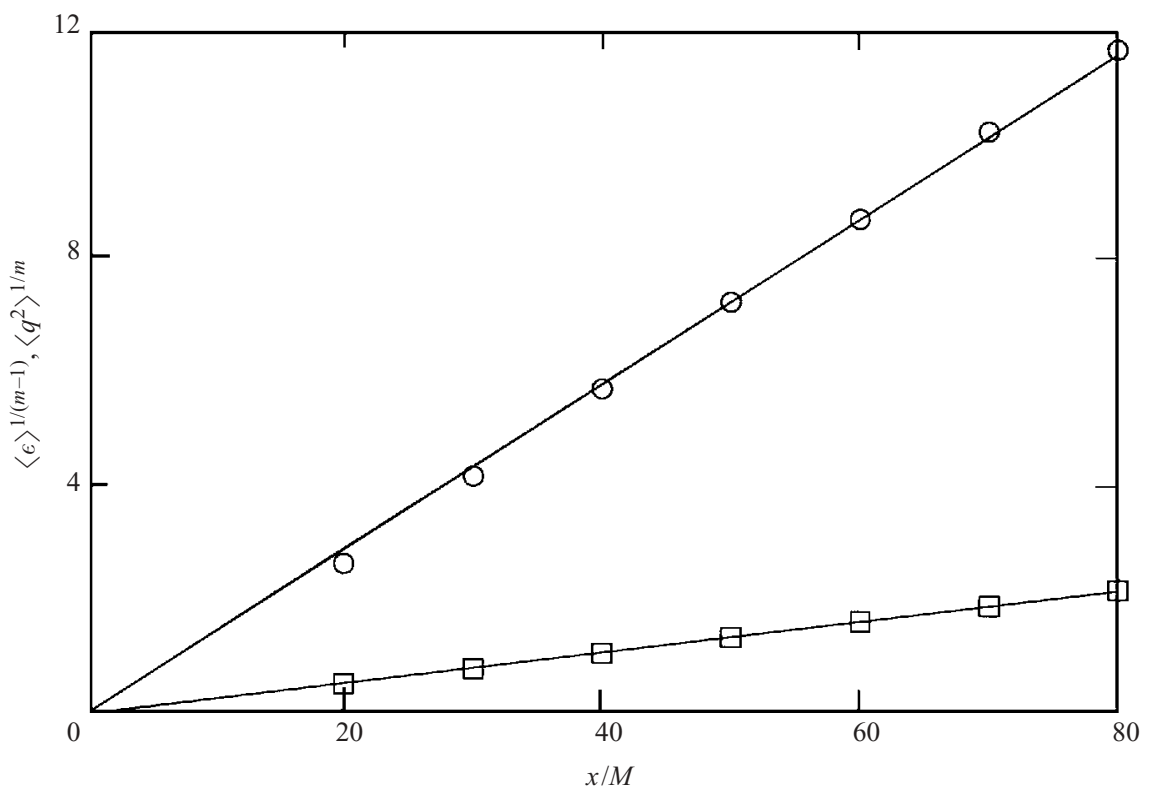

FIGURE 3. Streamwise variation of $\left\langle q^{2}\right\rangle^{1 / m}$ and $\langle\epsilon\rangle^{1 /(m-1)}$, with $m=-1.25 .\left\langle q^{2}\right\rangle$ is in $\mathrm{m}^{2} \mathrm{~s}^{-2}$ and $\langle\epsilon\rangle$ is $\mathrm{m}^{2} \mathrm{~s}^{-3} \cdot \circ,\left\langle q^{2}\right\rangle^{1 / m} ; \square,\langle\epsilon\rangle^{1 /(m-1)}$. The straight lines through the origin are least-squares regressions to the data.

$30 \leqslant x / M \leqslant 80$ occurs when $x_{0} \simeq 0$. Using (2.17) and the value corresponding to the horizontal line in figure 2 yields $m \simeq-1.25$. The variation of $\left\langle q^{2}\right\rangle^{1 / m}$ and $\langle\epsilon\rangle^{1 / m-1}$ with $\left(x-x_{0}\right) / M$ is shown in figure 3 for $m=-1.25$ and the best choice for $x_{0}(\simeq 0)$. 


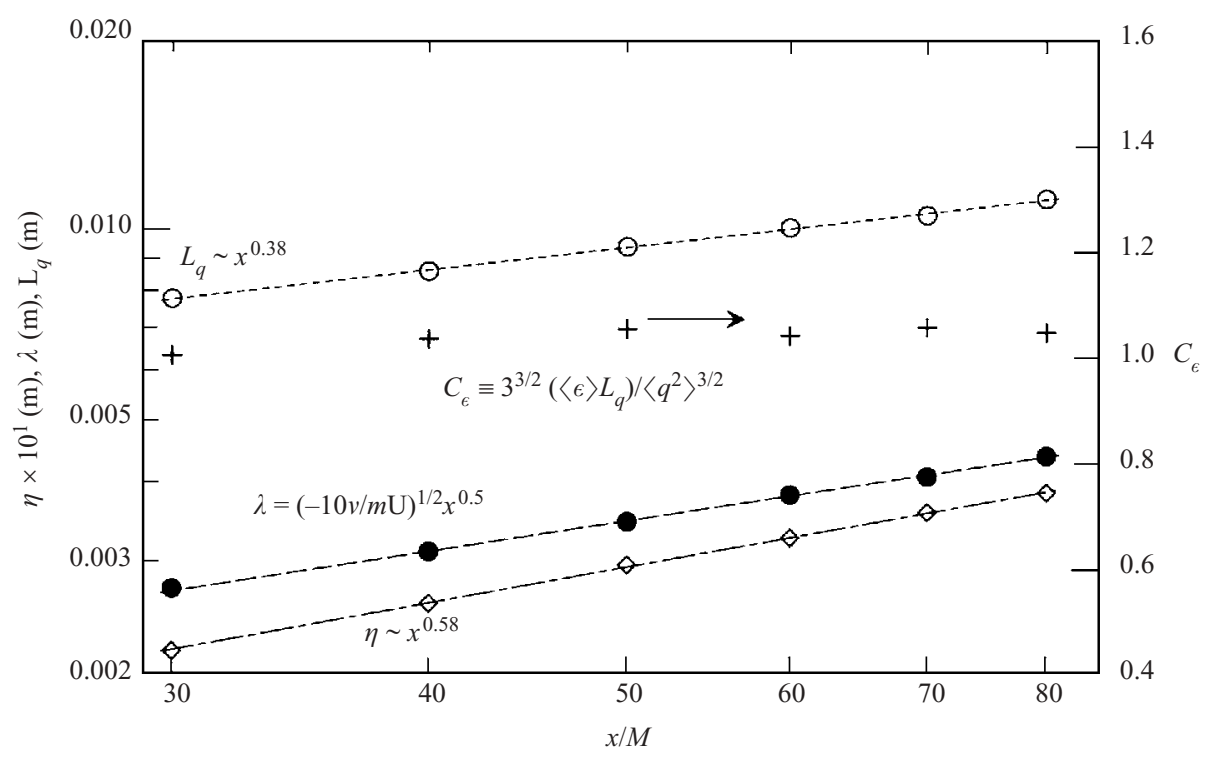

FIGURE 4. Comparison between streamwise growth rates of the integral length scale $L_{q}$, the Taylor microscale $\lambda$ and the Kolmogorov length scale $\eta$. The non-dimensional mean energy dissipation rate $C_{\epsilon}$ is shown on the right ordinate. $\diamond, \eta ; \bullet, \lambda ; \bigcirc, L_{q} ;+, C_{\epsilon}$.

The straight lines which go through the origin represent the data adequately over the range $30 \leqslant\left(x-x_{0}\right) / M \leqslant 80$, allowing for the uncertainty (\$3) in $\left\langle q^{2}\right\rangle$ and $\langle\epsilon\rangle$. Since $x_{0} \simeq 0$ in the present experiment, $x$, instead of $\left(x-x_{0}\right)$, will be used in subsequent plots.

The integral length scale is defined by

$$
L=\frac{\pi}{2\left\langle q^{2}\right\rangle} \int_{0}^{\infty} \frac{E(k)}{k} \mathrm{~d} k
$$

In experiments, $E(k)$ is difficult to estimate; in any case, it is almost invariably estimated from the one-dimensional spectrum using an assumption (isotropy) that is unlikely to be valid at small $k$. A definition, based on the velocity autocorrelation function, is usually adopted:

$$
L_{\alpha}=\frac{1}{\left\langle\alpha^{2}\right\rangle} \int_{0}^{r_{\alpha}} B_{\alpha, \alpha}(r) \mathrm{d} r
$$

where $B_{\alpha, \alpha}$ is the correlation $\langle\alpha(x) \alpha(x+r)\rangle$, with $\alpha \equiv u$, $v$ or $w$. For $\alpha \equiv q$, $B_{q, q} \equiv\langle u(x) u(x+r)\rangle+\langle v(x) v(x+r)\rangle+\langle w(x) w(x+r)\rangle$. The limit $r_{\alpha}$ corresponds to the first zero-crossing of $B_{\alpha, \alpha}(r)$. Irrespective of whether (4.1) or (4.2) $(\alpha \equiv q)$ is adopted, similarity requires that $L$ (or $L_{q}$ ) $\sim \lambda \sim x^{1 / 2}$ (George et al. 2001; Wang \& George 2002). This can be readily established after dividing $L$ and $L_{q}$ by $\lambda$, i.e.

$$
\tilde{L} \equiv \frac{L}{\lambda}=\frac{\pi}{2} \int_{0}^{\infty} \frac{\tilde{E}(\tilde{k})}{\tilde{k}} \mathrm{~d} \tilde{k} \quad \tilde{L}_{q} \equiv \frac{L_{q}}{\lambda}=\int_{0}^{\tilde{r}_{q}} \tilde{B}_{q, q}(\tilde{r}) \mathrm{d} \tilde{r},
$$

where $\tilde{k}=k \lambda, \tilde{E}(\tilde{k})=\lambda E(k) /\left\langle q^{2}\right\rangle$ (in general, a tilde will denote normalization by $\lambda$ and /or $\left.\left\langle q^{2}\right\rangle\right)$. However, the present estimates of $L_{q}$, with $B_{\alpha, \alpha} \equiv 1-\left(\left\langle(\delta \alpha)^{2}\right\rangle\right) / 2\left\langle\alpha^{2}\right\rangle$, indicate that it is not proportional to $\lambda . L_{q}$ varies proportionately to $\left(x-x_{0}\right)^{0.38}$, as shown in figure 4 . The streamwise variations of $L_{u}, L_{v}, L_{w}$ and $L_{q}$, normalized by the 


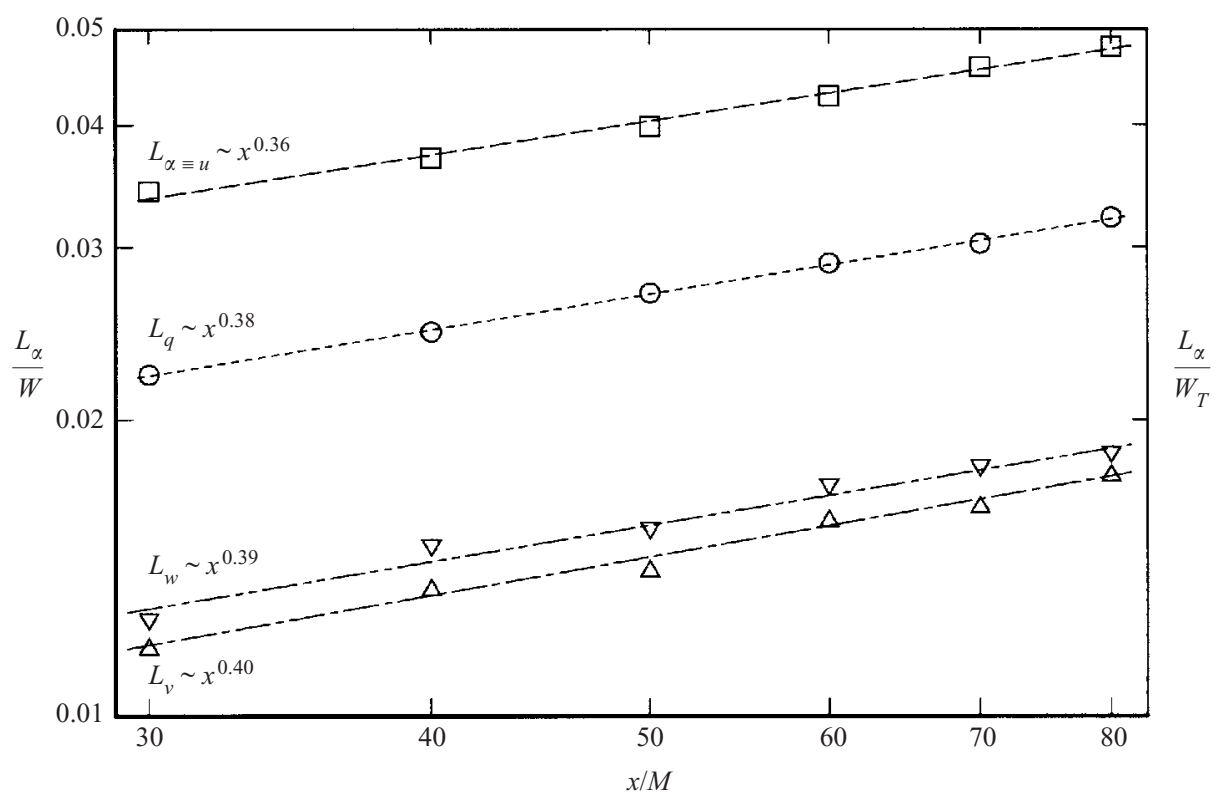

FIGURE 5. Streamwise variation of the integral length scale $L_{\alpha}$ associated with different velocity fluctuations and normalized by $W$, the transverse dimension of the wind tunnel working section. $\square, \alpha \equiv u ; \Delta, v ; \nabla, w ; \bigcirc, q$.

tunnel lateral dimension, $W(=350 \mathrm{~mm})$, are given in figure 5 . The decay exponents associated with these three length scales are of the same order as the exponent for $L_{q}$. Specifically, least-squares fits to the data suggest that $L_{u} \sim\left(x-x_{0}\right)^{0.36}, L_{v} \sim\left(x-x_{0}\right)^{0.40}$ and $L_{w} \sim\left(x-x_{0}\right)^{0.39}$. The power-law dependence for $L_{q}$ is also consistent with that determined by assuming that the non-dimensional energy dissipation rate $C_{\epsilon}$ $\left[\equiv 3^{3 / 2}\langle\epsilon\rangle L_{\epsilon}\left\langle q^{2}\right\rangle^{-3 / 2}\right]$ is constant (e.g. Batchelor 1953; Tennekes \& Lumley 1972). Sreenivasan (1984) indicated that $C_{\epsilon}$ was a flow-dependent constant (equal to $\simeq 1$ for grid turbulence) when $R_{\lambda} \gtrsim 50$. Antonia \& Pearson (2000) (also Antonia, Zhou \& Romano 2002b) have shown that, for nominally the same flow (a two-dimensional wake) and $R_{\lambda}$, the magnitude of $C_{\epsilon}$ may vary significantly when the initial conditions are varied. The difficulty in estimating $L_{\epsilon}$ is that $C_{\epsilon}$ is not known a priori. To estimate $C_{\epsilon}$ here, we have identified $L_{\epsilon}$ with $L_{q}$. The present data for $L_{q},\langle\epsilon\rangle$ and $\left\langle q^{2}\right\rangle$ yield a value of $C_{\epsilon}$ equal to $1.03 \pm 0.03$ (see figure 4). For the $M=5.08 \mathrm{~cm}$ square rod grid of Comte-Bellot \& Corrsin (1971), $C_{\epsilon}^{u} \equiv\langle\epsilon\rangle L_{f}\left\langle u^{2}\right\rangle^{-3 / 2}$, where $L_{f}$ is the longitudinal integral length scale based on two-point space-time correlations, and is equal to 1.04 at $x / M=42$ and $x / M=98$. For these data (see Comte-Bellot \& Corrsin 1966), $L_{f} \sim\left(x-x_{0}\right)^{0.35}$ and $R_{\lambda} \sim\left(x-x_{0}\right)^{-0.15}$; the present data for $L_{u}$ and $R_{\lambda}$ agree closely with these variations. Constancy of $C_{\epsilon}$ implies that $L_{\epsilon} \sim\left(x-x_{0}\right)^{(2+m) / 2}$; with $m=-1.25$, $L_{\epsilon} \sim\left(x-x_{0}\right)^{0.35}$. Comte-Bellot \& Corrsin (1966) reported that their transverse integral length scale $L_{g}$ varied as $\left(x-x_{0}\right)^{0.40}$; the present growth rate of $L_{v}$ is in accord with this.

At $x / M=80, L_{q} / W \sim 0.032, L_{u} / W \simeq 0.048, L_{v} / W \simeq 0.017$ and $L_{w} / W \simeq 0.018$. Whilst these values appear to be small, the maximum distance over which correlation can exist is likely to be larger than the integral length scale (this is discussed further in $\S 5$, where the behaviour of $\left\langle(\delta q)^{2}\right\rangle$ is considered). Corrsin's 


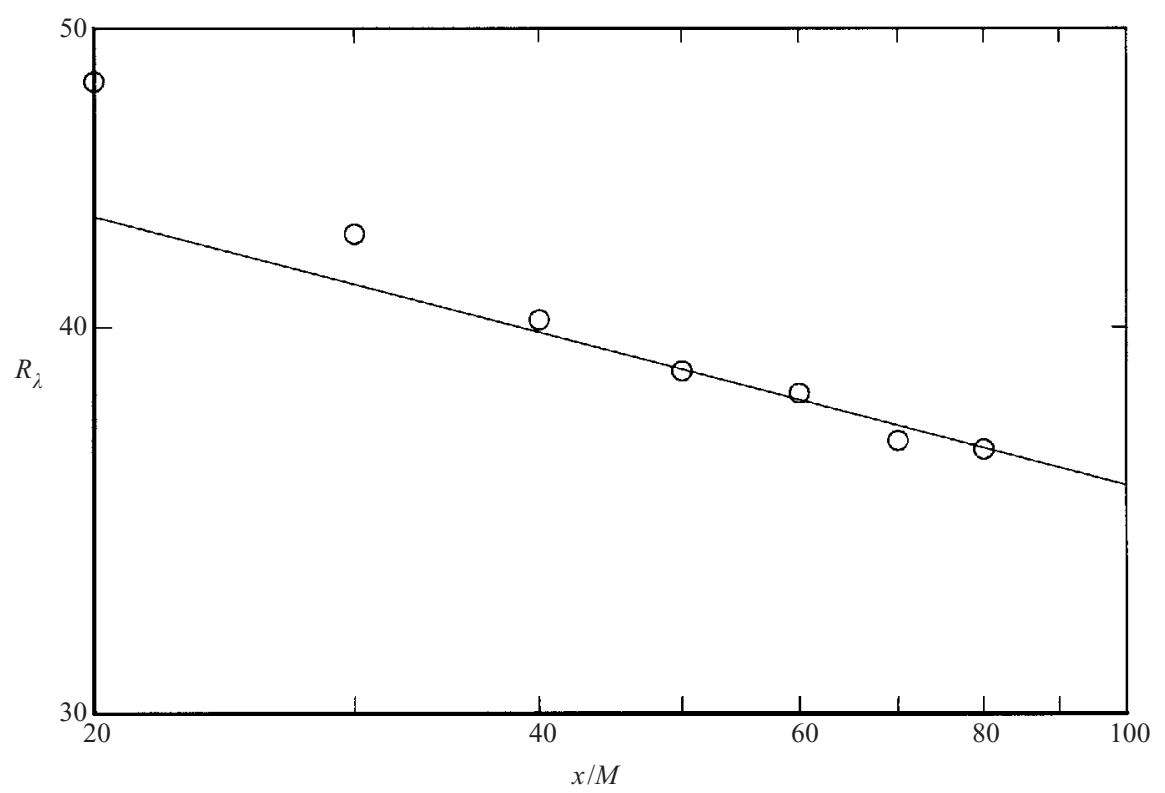

FIGURE 6. Streamwise variation of $R_{\lambda}$. The straight line is derived from the fit to $\left\langle q^{2}\right\rangle^{1 / m}$ in figure 3 and (2.17). $\bigcirc, R_{\lambda} ;-, 63.2(x / M)^{-0.125}$.

(1963) criterion that $W$ "should be much larger than the maximum distance over which some correlation can be detailed" is unlikely to be satisfied adequately. George et al. (2001) and Wang \& George (2002) have shown that the ratio $L / \lambda$ is not constant for DNS decaying turbulence data in a periodic box. Only after a significant correction is applied to the low-wavenumber part of $E(k)$ does the ratio $L / \lambda$ become constant, in agreement with G92 and the present analysis.

The ratios $L_{u} / L_{v}$ and $L_{u} / L_{w}$ are 2.75 and 2.55 , significantly larger than the isotropic value of 2 . Another measure of anisotropy is provided by the ratios $\left\langle v^{2}\right\rangle /\left\langle u^{2}\right\rangle$ and $\left\langle w^{2}\right\rangle /\left\langle u^{2}\right\rangle$ (for isotropic turbulence, these ratios should be equal to 1). In the present experiments, $\left\langle v^{2}\right\rangle /\left\langle u^{2}\right\rangle \simeq 0.72$ and $\left\langle w^{2}\right\rangle /\left\langle u^{2}\right\rangle \simeq 0.88$. While the previous length scale and turbulence intensity ratios suggest that the large scales deviate from isotropy, it should also be recognized that the small scales only satisfy isotropy approximately. Antonia et al. (1998) indicated that the components of $\langle\epsilon\rangle$ exhibit non-negligible departures from isotropy, although $\langle\epsilon\rangle_{\text {iso }}$ and $\langle\epsilon\rangle_{\text {hom }}$ approximate $\langle\epsilon\rangle$ adequately, due to compensations in these departures.

In view of the above-mentioned departures from isotropy, the usual definition of $R_{\lambda} \equiv\left\langle u^{2}\right\rangle^{1 / 2} \lambda / v$ with $\lambda \equiv\left\langle u^{2}\right\rangle^{1 / 2} /\left\langle(\partial u / \partial x)^{2}\right\rangle^{1 / 2}$ is not used here. Instead, a more general definition, (2.18), with $\lambda$ given by (2.16), is used. This definition avoids the ambiguity (e.g. Corrsin 1963; Fulachier \& Antonia 1983) arising from the directional dependence of $\left\langle u^{2}\right\rangle$ and $\left\langle u^{2}\right\rangle /\left\langle(\partial u / \partial x)^{2}\right\rangle$. For isotropic turbulence, $\left\langle q^{2}\right\rangle=3\left\langle u^{2}\right\rangle$ and $\langle\epsilon\rangle=15 v\left\langle(\partial u / \partial x)^{2}\right\rangle$, so that the present definitions of $\lambda$ and $R_{\lambda}$ coincide with those commonly used in the literature. From (2.17) and (2.15), it follows that $R_{\lambda}$ varies proportionately to $\left(x-x_{0}\right)^{(m+1) / 2}$ and must therefore decrease with $x$, albeit slowly. The measured values of $R_{\lambda}$ (figure 6) are consistent with a $\left(x-x_{0}\right)^{-0.125}$ decay only over the range $40 \leqslant x / M \leqslant 80$. 

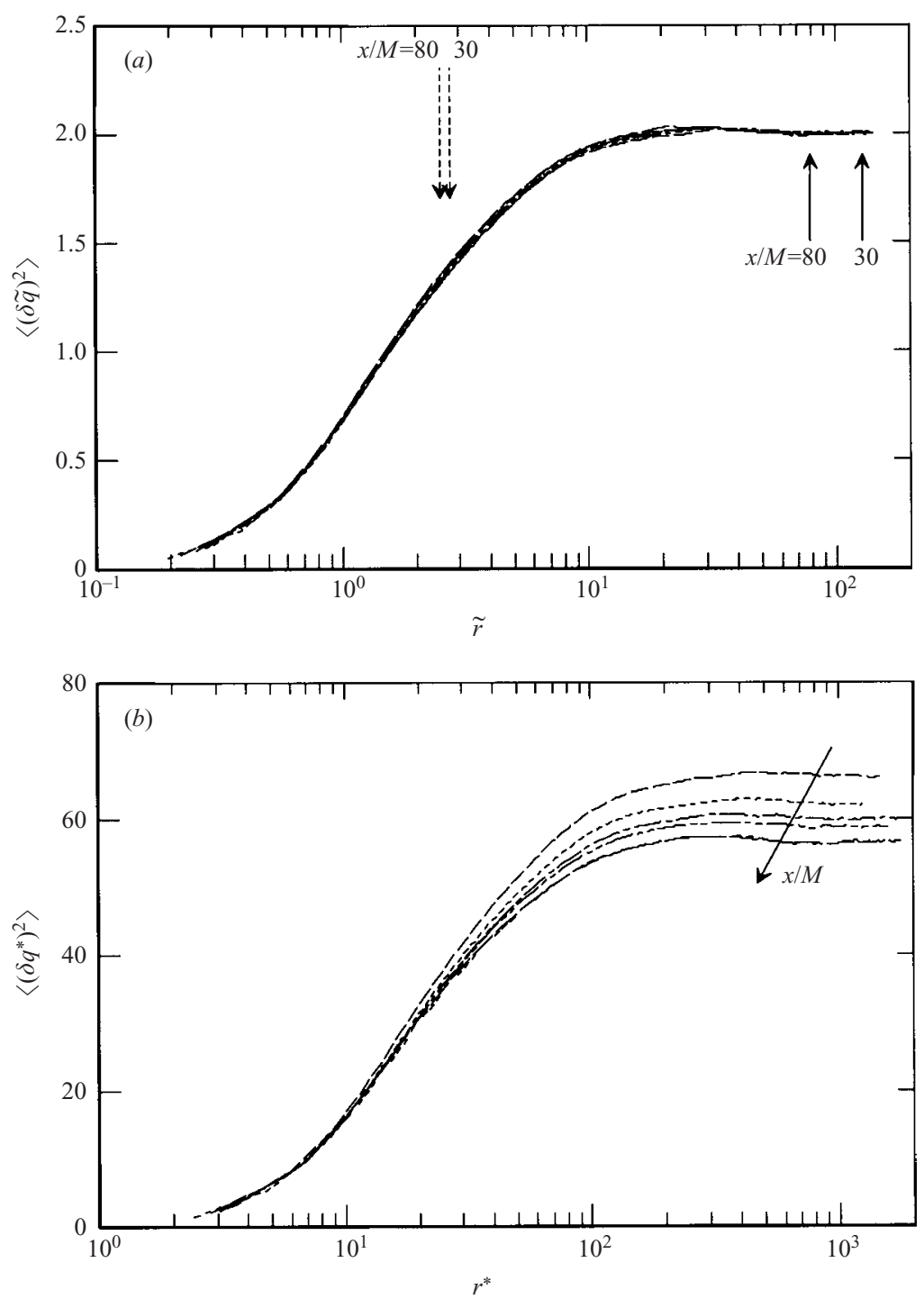

FiguRE 7. Turbulent energy structure function normalized using $(a)\left\langle q^{2}\right\rangle$ and $\lambda,(b) U_{K}^{2}$ and $\eta$. -- --, $x / M=30 ;-\cdots, 40 ;-\cdots, 50 ;-\cdots, 60 ;-\cdots,--70 ;-\ldots, 80$. The two solid arrows in $(a)$ indicate the range of values for $W / \lambda$ between $x / M=30$ and $x / M=80$; the broken arrows indicate the corresponding range of values for $L_{\alpha} / \lambda$. In $(b)$, the arrow is in the direction of increasing $x / M$.

\section{Similarity of second- and third-order structure functions}

The structure function $\left\langle(\delta q)^{2}\right\rangle$ was constructed from the sum of $\left\langle(\delta u)^{2}\right\rangle,\left\langle(\delta v)^{2}\right\rangle$ and $\left\langle(\delta w)^{2}\right\rangle$. Digital time series for $u, v, w$ were used to form the temporal increments

$$
\delta u=u(t+\tau)-u(t), \quad \delta v=v(t+\tau)-v(t), \quad \delta w=w(t+\tau)-w(t)
$$

for a range of values of $\tau$ (the smallest value of $\tau$ corresponds to one sample, equal to $f_{s}^{-1}$ ). These increments may be interpreted directly as spatial increments by using Taylor's hypothesis (with $r \equiv U \tau$ ). Distributions of $\left\langle(\delta \tilde{q})^{2}\right\rangle$ are shown in figure 7(a) as a function of $\tilde{r}$ for $30 \leqslant x / M \leqslant 80$. The collapse is adequate for nearly all values 
of $\tilde{r}$ (note that a linear scale is used for the ordinate). Arguably, the quality of the collapse appears to be best when $\tilde{r}$ is either approximately 1 or exceeds 30 . This latter feature is a consequence of using $\left\langle q^{2}\right\rangle$ for normalization: for large $\tilde{r},\left\langle(\delta \tilde{q})^{2}\right\rangle$ must equal 2 when the velocity fluctuations become decorrelated and local streamwise homogeneity is assumed. At very small $\tilde{r}$, all the data closely satisfy the expectation $\left\langle(\delta \tilde{q})^{2}\right\rangle \sim \tilde{r}^{2}$. The solid vertical arrows in figure $7(a)$ give a more realistic indication of the values of $\tilde{r}$, for the relevant range of $x / M$, which may be affected by the dimension $W$ of the tunnel working section than the ratios $L_{\alpha} / W$ (figure 5). For $x / M=80$, the arrow occurs only just beyond the value of $\tilde{r}$ where $\left\langle(\delta \tilde{q})^{2}\right\rangle$ becomes constant. The possibility that the largest scales may be affected by the spatial constraints of the tunnel at $x / M=80$ cannot be ruled out. The ratio $L_{\alpha} / \lambda$ is also shown in figure $7(a)$ as vertical broken arrows. It is evident that this ratio is significantly smaller than the value of $\tilde{r}$ at which $\left\langle(\delta \tilde{q})^{2}\right\rangle$ becomes constant so that the magnitude of $L_{\alpha} / W$ (figure 5) does not provide a sensitive enough measure of the influence of the tunnel boundaries.

For comparison, $\left\langle(\delta q)^{2}\right\rangle$ is plotted in figure $7(b)$ using Kolmogorov scaling, i.e. $\left\langle\left(\delta q^{*}\right)^{2}\right\rangle \equiv\left\langle(\delta q)^{2}\right\rangle / U_{K}^{2}$ and $r^{*} \equiv r / \eta$. While there is relatively good collapse for $r^{*} \leqslant 10$, i.e. over the dissipative range, there is a clearly discernible $R_{\lambda}$ dependence in $\left\langle\left(\delta q^{*}\right)^{2}\right\rangle$ for $r^{*} \geqslant 10^{3}$. Using the definitions of $\lambda$ and $R_{\lambda}$, the limiting value of $\left\langle\left(\delta q^{*}\right)^{2}\right\rangle$, when $r^{*} \rightarrow \infty$, is given by (e.g. Antonia 2000)

$$
\left\langle\left(\delta q^{*}\right)^{2}\right\rangle=\frac{2}{15^{1 / 2}}\left(1+\frac{\left\langle v^{2}\right\rangle}{\left\langle u^{2}\right\rangle}+\frac{\left\langle w^{2}\right\rangle}{\left\langle u^{2}\right\rangle}\right) R_{\lambda} .
$$

In isotropic turbulence, $\left\langle v^{2}\right\rangle /\left\langle u^{2}\right\rangle=\left\langle w^{2}\right\rangle /\left\langle u^{2}\right\rangle=1$, so that

$$
\left\langle\left(\delta q^{*}\right)^{2}\right\rangle=\frac{6 R_{\lambda}}{15^{1 / 2}} .
$$

The magnitude of this limiting value must decrease with $x$ since $R_{\lambda}$ decreases with $x$ (figure 6). The data at large $r^{*}$ in figure $7(b)$ are consistent with this. One characteristic of the flow downstream of the present grid (round rods) is that $\left\langle(\delta \tilde{q})^{2}\right\rangle$ overshoots 2 before becoming constant at large $\tilde{r}$. An equivalent undershoot (below zero) exists for $B_{q, q}$, since $B_{q, q}(r)=\left\langle q^{2}\right\rangle-\left\langle(\delta q)^{2}\right\rangle / 2$. This behaviour is unlikely to be universal, since the shape of $\left\langle(\delta q)^{2}\right\rangle$ (or $B_{q, q}(r)$ ) depends on the conditions at the grid. There is no overshoot when the grid is constructed from square rods with the same characteristic dimensions (and blockage ratio) as for the present grid. Although this may not be surprising, it does suggest that during the initial period of decay, the effect of initial conditions on the large scales may be significant, at least for the present small values of $R_{\lambda}$. This, in turn, implies that the effect of initial conditions on smaller scales, including those which belong to the dissipative range, may also not be negligible.

There seems to be little doubt, when comparing figure $7(a)$ with figure $7(b)$, that, overall, scaling on $\left\langle q^{2}\right\rangle$ and $\lambda$ is superior to that based on Kolmogorov variables. The latter scaling is adequate only for dissipative scales, typically $r^{*} \lesssim 10$; it is however expected (e.g. Zhou \& Antonia 2000) that this range will expand as $R_{\lambda}$ increases. In the limit $r^{*} \rightarrow 0$, both $\lambda$-based and Kolmogorov normalization are compatible since it can be readily shown that

$$
\lim _{r^{*} \rightarrow 0} \frac{\left\langle\left(\delta q^{*}\right)^{2}\right\rangle}{r^{* 2}}=\frac{1}{3}
$$



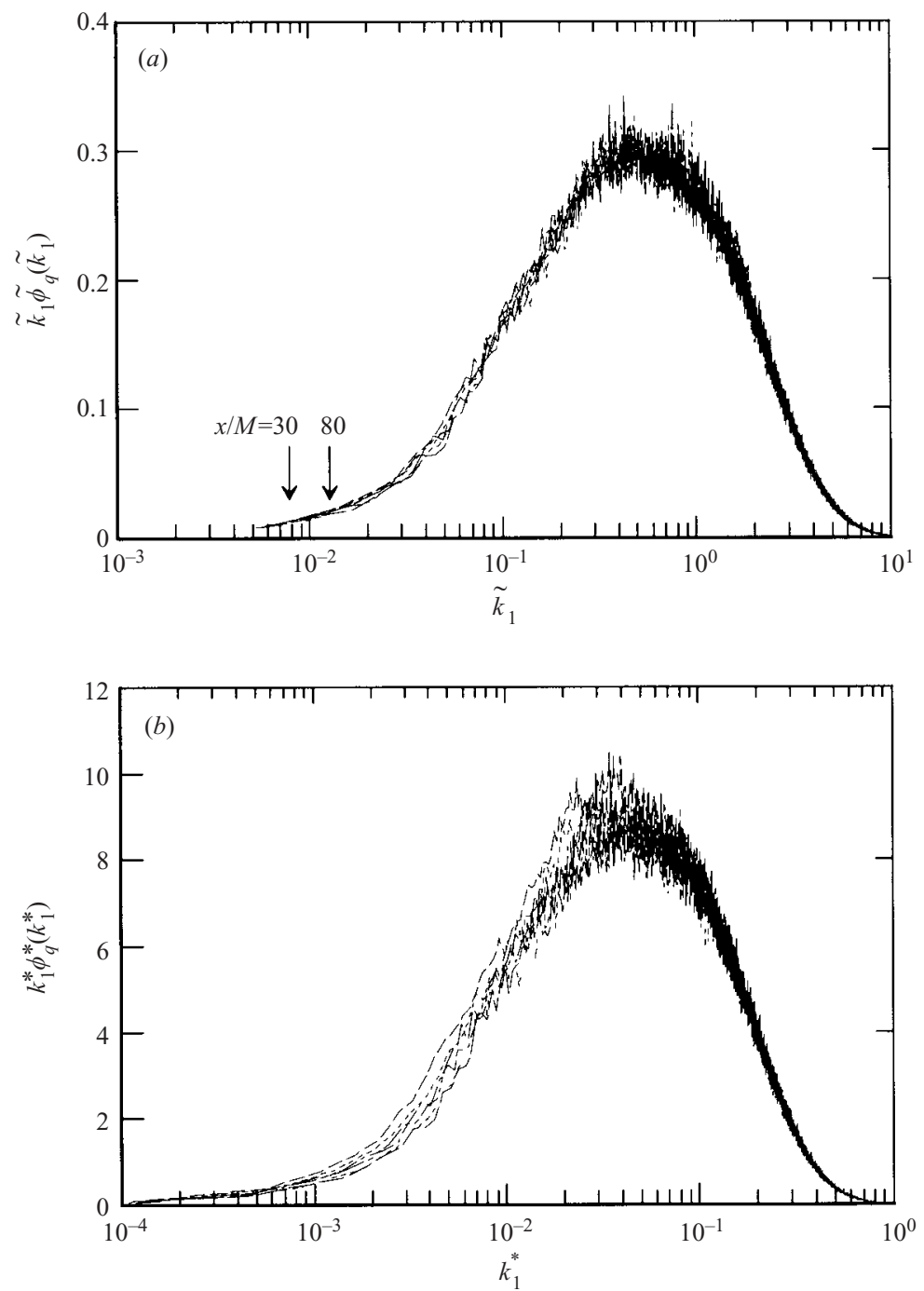

FiguRE 8. Turbulent energy spectra normalized using $(a)\left\langle q^{2}\right\rangle$ and $\lambda,(b) U_{K}^{2}$ and $\eta$. Symbols are as in figure 7. The two arrows in $(a)$ indicate the range of $\lambda / W$ between $x / M=30$ and $x / M=80$.

and

$$
\lim _{\tilde{r} \rightarrow 0} \frac{\left\langle(\delta \tilde{q})^{2}\right\rangle}{\tilde{r}^{2}}=\frac{5}{3}
$$

The equally good collapse at small $\tilde{r}$ or small $r^{*}$ in figures $7(a)$ and $7(b)$ respectively is therefore not surprising.

For completeness, we also present the spectrum corresponding to $\left\langle q^{2}\right\rangle$, i.e.

$$
\phi_{q}\left(k_{1}\right)=\phi_{u}\left(k_{1}\right)+\phi_{v}\left(k_{1}\right)+\phi_{w}\left(k_{1}\right),
$$

where the spectral density $\phi_{\alpha}\left(k_{1}\right)$ is such that $\int_{0}^{\infty} \phi_{\alpha}\left(k_{1}\right) \mathrm{d} k_{1}=\left\langle\alpha^{2}\right\rangle \quad(\alpha \equiv u, v, w$ or $q)$ and $k_{1}(\equiv 2 \pi f / U)$ is the one-dimensional wavenumber. When $\left\langle q^{2}\right\rangle$ and $\lambda$ are used for normalization (figure $8 a), \tilde{\phi}_{q}\left(\tilde{k}_{1}\right)$ is such that $\int_{0}^{\infty} \tilde{\phi}_{q}\left(\tilde{k}_{1}\right) \mathrm{d} \tilde{k}_{1}=1$; for 
Kolmogorov-normalization (figure $8 b), \int_{0}^{\infty} \phi_{q}^{*}\left(k_{1}^{*}\right) \mathrm{d} k_{1}^{*}=\left\langle q^{2}\right\rangle / U_{K}^{2}$. This latter quantity varies proportionately to $R_{\lambda}$ since, for isotropic turbulence, $\left\langle q^{* 2}\right\rangle=3 R_{\lambda} / 15^{1 / 2}$. The semi-log plot of figure $8(a)$ highlights the low-wavenumber energy-containing range, yet preserves the integral area. There is no loss of information with respect to testing similarity. Comparison between figure $8(a)$ and figure $8(b)$ shows once again that there is better collapse when $\left\langle q^{2}\right\rangle$ and $\lambda$ are used. Since the maximum energy occurs at $\tilde{k}_{1} \simeq 0.5$ (over the present range of $R_{\lambda}$ ), $\lambda$ represents adequately the part of the spectrum which contributes significantly to the energy. Although there is scatter in the distributions of $\tilde{k}_{1} \tilde{\phi}_{q}\left(\tilde{k}_{1}\right)$ around $\tilde{k}_{1}=0.5$, this is smaller than that around the peak value $\left(k_{1}^{*} \simeq 0.04\right)$ when Kolmogorov normalization is used. The two vertical arrows in figure $8(a)$ indicate the range of values of $W^{-1} \lambda$ corresponding to $30 \leqslant x / M \leqslant 80$. Although the location $\left(\tilde{k}_{1} \simeq 0.5\right)$ of the peak in $\tilde{k}_{1} \tilde{\phi}_{q}\left(\tilde{k}_{1}\right)$ is comfortably larger than the largest value of $W^{-1} \lambda$, the possibility that the boundaries of the wind tunnel's working section may have affected the largest scales in the flow cannot be dismissed.

G92 showed that the spectra measured by Comte-Bellot \& Corrsin (1971) were consistent with the similarity solution admitted by the spectral energy equation, (1.3). The one-dimensional spectra of Comte-Bellot \& Corrsin are plotted here (figure 9) using the same presentation as in figure 8 . The use of a linear ordinate is more likely to show departures from similarity than the logarithmic scale used in G92. The range of $x / M$ (45 to 385) used by Comte-Bellot \& Corrsin is significantly larger than in the present study although the magnitude and percentage variation (48.6 to 366) are comparable to those in the present study. The distributions in figure 9 indicate little difference between the two types of scaling when $\tilde{k}_{1}$ and $k_{1}^{*}$ are large. At small wavenumbers, the systematic dependence on $x / M$ (or $R_{\lambda}$ ) in figure $9(b)$ is not discernible in figure $9(a)$. Nonetheless, the data display significant scatter and the quality of collapse in figure $8(a)$ is superior and supports G92 and the present theory $(\S 2)$ better than figure $9(a)$.

We now turn our attention to the third-order structure function $\left\langle(\delta u)(\delta q)^{2}\right\rangle$, equal to the sum $\left(\left\langle(\delta u)^{3}\right\rangle+\left\langle(\delta u)(\delta v)^{2}\right\rangle+\left\langle(\delta u)(\delta w)^{2}\right\rangle\right)$. When the normalization uses $\left\langle q^{2}\right\rangle$ and $\lambda$, the collapse is not as good as in figure $7(a)$. The magnitude of $g\left(\equiv-3^{1 / 2} R_{\lambda}\left\langle(\delta \tilde{u})(\delta \tilde{q})^{2}\right\rangle\right)$ at $x / M=30$ is significantly larger for $\tilde{r} \lesssim 5$ than that of all the other distributions. It is however reasonable to assume that $x / M=30$ may be insufficient for the third-order structure function to satisfy similarity; the $R_{\lambda}$ plot of figure 6 indeed suggests that a power-law behaviour for $R_{\lambda}$ is obtained only when $x / M$ exceeds 30 . It is further possible that the distribution at $x / M=80$ may have been affected by the tunnel boundaries. In the light of the previous remarks, the collapse of the results in the range $40 \leqslant x / M \leqslant 70$ is probably tolerable, given the uncertainly in determining $g$ (see figure 11). The quality of collapse in figure $10(b)$ is only marginally worse than for figure 10(a); as expected, the curves overlap satisfactorily when $r^{*} \lesssim 10$. Although the measured $g$ (figure 10a) satisfies (2.2) to a lesser degree than the measured $f$ (figure 7a) satisfies (2.1), it is plausible that the third-order structure function represents a more sensitive, if not stringent, test of local isotropy than the second-order structure function. One would expect $\left\langle(\delta u)(\delta q)^{2}\right\rangle$ to be measured less accurately than $\left\langle(\delta q)^{2}\right\rangle$; an indirect measure of the uncertainty in $\left\langle(\delta \tilde{u})(\delta \tilde{q})^{2}\right\rangle$ is the scatter (figure 10) at large $\tilde{r}$ or $r^{*}$ when $\left\langle(\delta \tilde{u})(\delta \tilde{q})^{2}\right\rangle$ should be zero. A better estimate of the uncertainty is to compare the scatter in the distribution of $\left\langle(\delta \tilde{u})(\delta \tilde{q})^{2}\right\rangle$ when formed from different combinations of the time series for $(\delta u),(\delta v)$ and $(\delta w)$ from the four X-wires.

Figure 11 shows one distribution of $g$ denoted by $g_{\text {meas }}$, at $x / M=50$. Also shown are dashed curves indicating the maximum scatter in all estimates of 

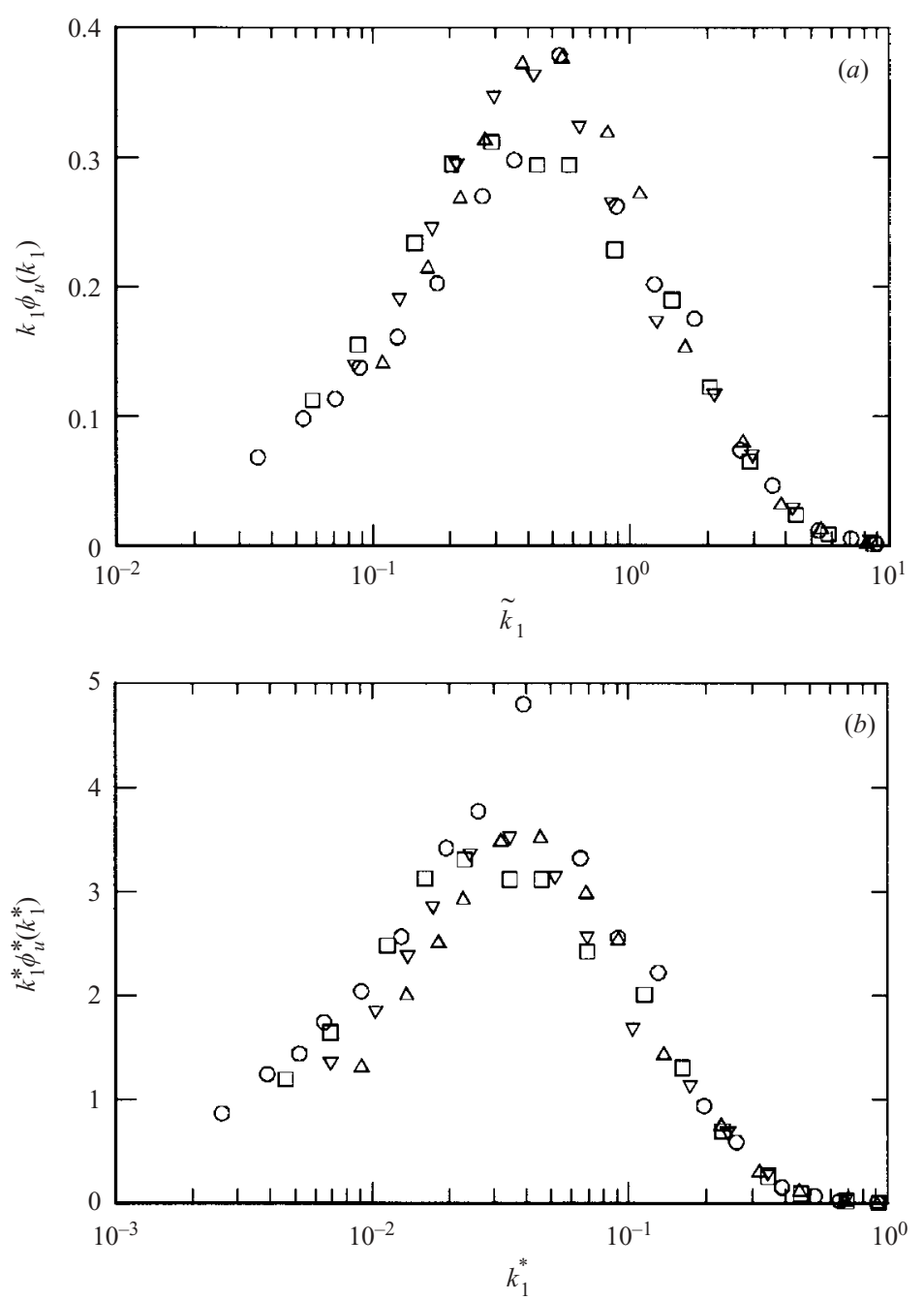

Figure 9. One-dimensional spectra of $u$ from Comte-Bellot \& Corrsin (1971) (2.54 cm grid; data are taken from table $2(b)$ of their paper): (a) normalization by $\lambda$ and $\left\langle q^{2}\right\rangle^{1 / 2} ;(b)$ normalization by $\eta$ and $U_{K} . \bigcirc, x / M=45 ; \square, 120 ; \nabla, 240 ; \triangle, 385$.

$-3^{1 / 2} R_{\lambda}\left\langle(\delta \tilde{u})(\delta \tilde{q})^{2}\right\rangle$ over $2 \leqslant \tilde{r} \leqslant 8$. Outside this region, all distributions of $g_{\text {meas }}$ exhibit relatively good collapse. Figure 11 also compares $g_{\text {meas }}$ with the calculation using (2.19), denoted by $g_{\text {cal }}$. The effect of neglecting the source term $\left(\Gamma_{1}\right)$ in $(2.19)$ is also indicated in this figure. The calculation, using all terms in (2.19), is most accurate between $\tilde{r} \simeq 1$ and approximately $2 L_{q}(\tilde{r} \simeq 5)$. A similar level of agreement was found at other values of $x / M$. Due to the numerical integration scheme used, the calculation does not extend to the smallest measured scale $(\tilde{r} \approx 0.27)$. However, the lack of resolution within this region is not of great consequence, since, as $\tilde{r} \rightarrow 0$, the dissipative term $f^{\prime}$ will be much larger than either the source terms (i.e. those containing $\Gamma_{1}$ and $\Gamma_{2}$ ) or $g$. The calculation carried out without the $\Gamma_{1}$ term is equivalent to that proposed by Danaila et al. (2002), in which their self-preserving form for $\left\langle(\delta q)^{2}\right\rangle$ yielded only the $\Gamma_{2}$ source term. As figure 11 shows, $\Gamma_{1}$ is required 

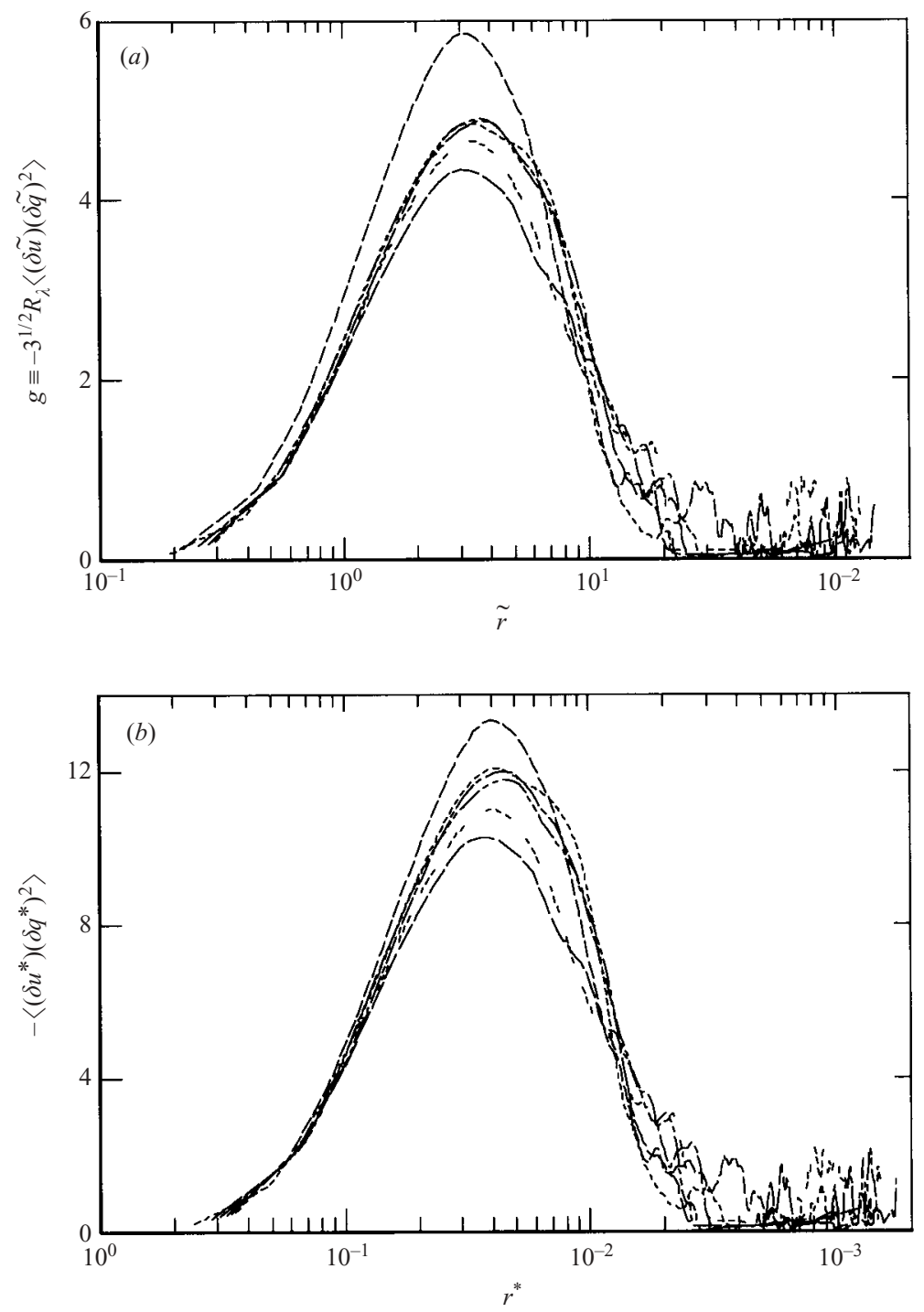

FIGURE 10. Third-order structure functions $-\left\langle(\delta u)(\delta q)^{2}\right\rangle$, normalized according to the present solution $(a)$ and Kolmogorov variables $(b)$. Symbols are as in figure 7.

for an accurate estimate of $g$. Ignoring $\Gamma_{1}$ and $\Gamma_{2}$, like Kolmogorov (1941b), would lead to an even greater error than that estimated (figure 11) by ignoring only $\Gamma_{1}$. For completeness, all terms in (2.19) are shown in figure 12. It is evident that the magnitude of the $\Gamma_{2}$ term increases rapidly as $\tilde{r}$ increases and dominates over all the other terms when $\tilde{r} \gtrsim 2$. The $\Gamma_{1}$ term is the next largest term for $\tilde{r} \gtrsim 7$. To allow comparison with figure 11, we have also included in figure 12 the contribution from $\left(g-5 \Gamma_{1} \tilde{r}^{-2} / m+10 \Gamma_{2} \tilde{r}^{-2}+2 f^{\prime}\right)$ (solid circles) and $\left(g+10 \Gamma_{2} \tilde{r}^{-2}+2 f^{\prime}\right)$ (open circles). The latter, where $\Gamma_{1}$ is ignored, does not provide as good an agreement with the line $20 \tilde{r} / 3$ than the former. It should however be noted that because of the log scale in figure 12 , the comparison between $g_{c a l}$ and $g_{\text {meas }}$ in figure 11 provides a more sensitive test for the balance of the terms in (2.19) than figure 12. 


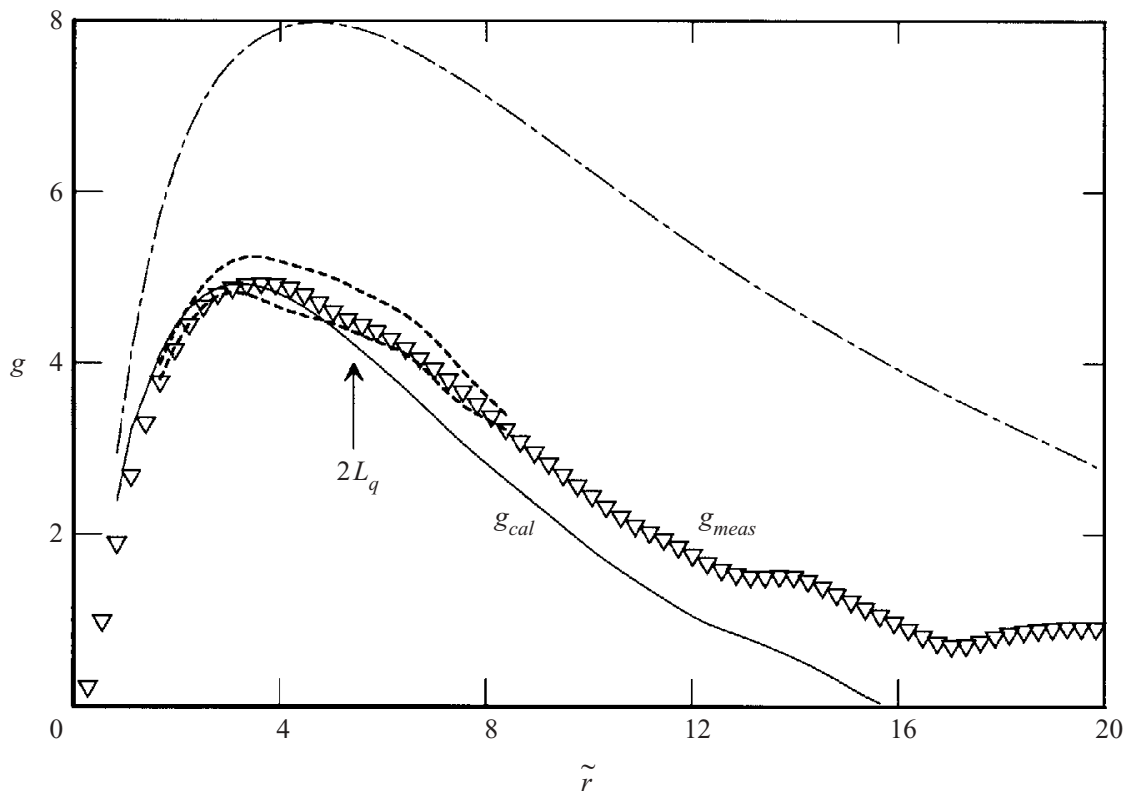

FIGURE 11. Comparison between measured and calculated distributions of $g$ (at $x / M=50$ ). $\nabla, g_{\text {meas }} ;-, g_{\text {cal }}$ using (2.19) and $m=-1.25$. The calculated curves start at a larger $\tilde{r}$ due to the numerical integration scheme used. - - - Maximum scatter in the estimate of $g_{\text {meas }}$ as determined from eight different combinations of $\delta u, \delta v$ and $\delta w$. The location of $2 L_{q}$ is indicated by an arrow. - - , $g_{\text {cal }}(2.19)$ with $\Gamma_{1}=0$.

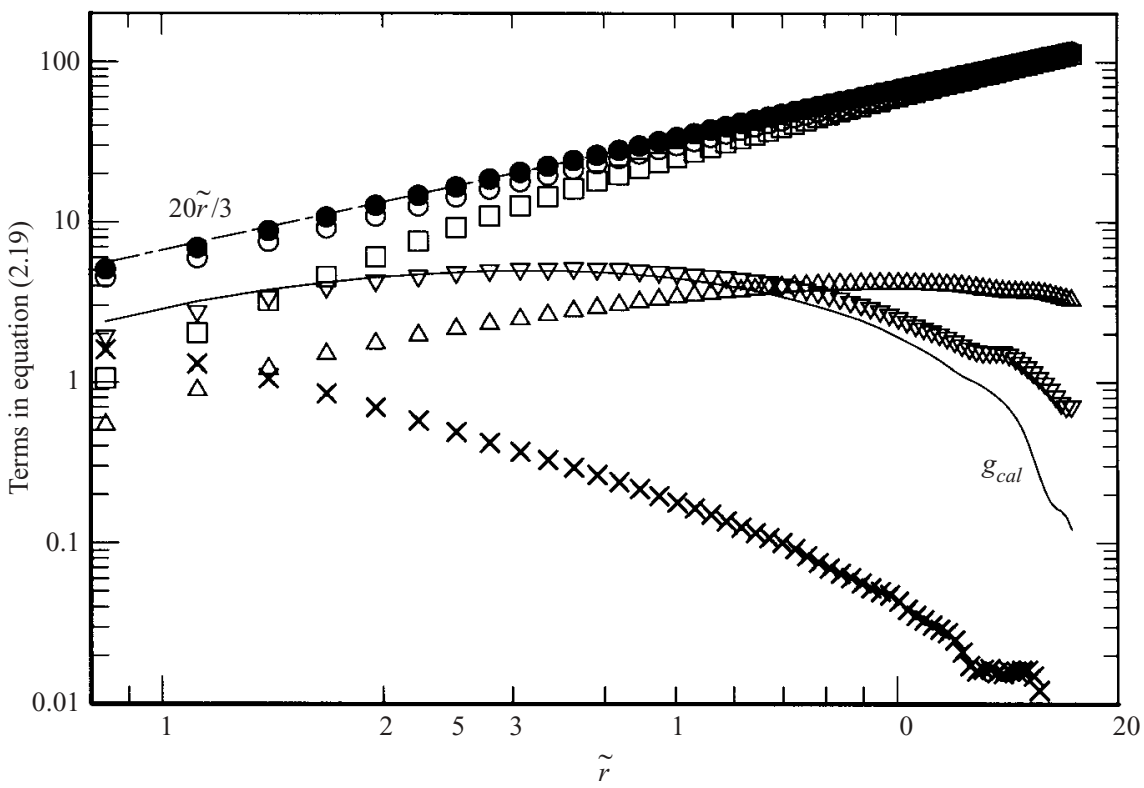

FigURE 12. Terms in (2.19) at $x / M=50 .--,(20 \tilde{r} / 3) ; \square, 10 \Gamma_{2} \tilde{r}^{-2} ; \triangle,-5 \Gamma_{1} \tilde{r}^{-2} / m ; \times, 2 f^{\prime}$; $\nabla,-3^{1 / 2} R_{\lambda}\left\langle(\delta \tilde{u})(\delta \tilde{q})^{2}\right\rangle ; \bullet,\left(g-5 \Gamma_{1} \tilde{r}^{-2} / m+10 \Gamma_{2} \tilde{r}^{2}+2 f^{\prime}\right) ; \bigcirc,\left(g+10 \Gamma_{2} \tilde{r}^{-2}+2 f^{\prime}\right) ;-, g_{\text {cal }}$. 


\section{Possible dependence of $f$ and $g$ on the Reynolds number}

The similarity solution given in $\S 2$ is valid strictly for fixed initial conditions. In particular, the relaxed scaling form of the third-order structure function yields a valid solution regardless of the magnitude of $R_{M}$. Both $f$ and $g$ will however vary as $R_{M}$ (which forms part of the initial conditions) is changed.

To illustrate the possible variation of $f$, we consider here a theoretical form for $f$, by parameterizing $\left\langle\left(\delta u^{*}\right)^{2}\right\rangle$ for decaying isotropic turbulence. The parameterization also allows a determination of the extent to which the source terms contribute to $g_{c a l}$ once $R_{M}$ (or more loosely $R_{\lambda}$ which will be used hereafter) becomes very large. Batchelor (1951) obtained an expression for $\left\langle\left(\delta u^{*}\right)^{2}\right\rangle$, strictly valid at large $R_{\lambda}$, for the dissipative (DR) and inertial (IR) ranges. It is explicitly assumed that there is no influence on the DR from the large scales. Stolovitzky, Sreenivasan \& Juneja (1993) modified Batchelor's parameterization to include the effect of internal intermittency in the IR, i.e. the possibility that the IR scaling exponent $\zeta_{u}$ may depart from its Kolmogorov (1941c) value of $2 / 3$. A more complete description of $\left\langle\left(\delta u^{*}\right)^{2}\right\rangle$ which extends to scales beyond the IR (and up to $L$ ) was obtained by Dhruva (2000) and subsequently used by Kurien \& Sreenivasan (2000). It is given by

$$
\left\langle\left(\delta u^{*}\right)^{2}\right\rangle=\frac{r^{* 2}\left(1+\beta r^{*}\right)^{(2 c-2)}}{15\left(1+\alpha r^{* 2}\right)^{c}},
$$

where $\alpha\left[\equiv 30^{(-3 / 2)}\right]$ is a measure of the cross-over between the DR and IR, $c \equiv 1-\zeta_{u} / 2$ and $\beta \equiv L^{*-1}$. To obtain an expression for $\left\langle\left(\delta q^{*}\right)^{2}\right\rangle$, the isotropic relation

$$
\left\langle\left(\delta q^{*}\right)^{2}\right\rangle=\left(3+r^{*} \frac{\mathrm{d}}{\mathrm{d} r^{*}}\right)\left\langle\left(\delta u^{*}\right)^{2}\right\rangle
$$

is used, giving

$$
\left\langle\left(\delta q^{*}\right)^{2}\right\rangle=\frac{r^{* 2}\left(1+r^{*} \beta\right)^{(2 c-3)}\left(5+2 \beta c r^{*}+3 \beta r^{*}-2 \alpha c r^{* 2}+5 \alpha r^{* 2}+3 \alpha \beta r^{* 3}\right)}{15\left(1+\alpha r^{* 2}\right)^{(c+1)}} .
$$

For isotropic turbulence, $\lambda^{*}=15^{1 / 4} R_{\lambda}^{1 / 2},\left\langle q^{* 2}\right\rangle=3 R_{\lambda} 15^{-1 / 2}$ and $L^{*} \equiv L_{\epsilon}^{*}=C_{\epsilon} R_{\lambda}^{3 / 2} 15^{-3 / 4}$. Relation (6.3) may be re-normalized by $\lambda$ and $\left\langle q^{2}\right\rangle$, yielding an expression for $\left\langle(\delta \tilde{q})^{2}\right\rangle$, denoted by $f_{p a r}$. Here $L_{\epsilon}$ is used with $C_{\epsilon}=1$, since the alternative definitions of $L$ ((4.1) and (4.2)) require a priori knowledge of $\left\langle(\delta u)^{2}\right\rangle$ for all $r$.

Figure 13 shows $f_{\text {par }}$ over a large $R_{\lambda}$ range $\left(100 \leqslant R_{\lambda} \leqslant 10^{6}\right)$ for $\zeta_{u}=2 / 3$. The inset in figure 13 compares (using a linear ordinate) a measured distribution of $f$ at $x / M=50\left(R_{\lambda} \simeq 40\right)$ with $f_{p a r}\left(\zeta_{u}=2 / 3\right)$. The agreement is best within the DR and when $\tilde{r}$ becomes large; evidently, the parameterization (6.3) fails to capture the effect of initial conditions. The discrepancy in the range $3<\tilde{r}<20$ is mainly associated with the effect of initial conditions of the grid, as was indicated in $\S 5$, and also the departure from isotropy in the experiment.

Using $f_{p a r}, g$ was calculated using (2.19); this calculation is denoted by $g_{p a r}$. According to Kolmogorov (1941b), $g_{\text {par }}$ should exhibit a $\tilde{r}$ behaviour within the IR at sufficiently large $R_{\lambda}$, with a pre-multiplier of 20/3. In the context of (2.19), this occurs when the contributions from the $\Gamma_{1}$ and $\Gamma_{2}$ terms are negligible. The $R_{\lambda}$ dependence of $g_{p a r} \tilde{r}^{-1}$ is shown in figure 13. The peak at small $R_{\lambda}$ begins to resemble a plateau at much higher $R_{\lambda}$. A separation between the energy-containing range and the DR may exist only when $R_{\lambda}$ exceeds about $10^{5}$; note that at $R_{\lambda} \simeq 10^{3}$, a magnitude achievable using active grids (e.g. Mydlarski \& Warhaft 1996), there is no evidence of 


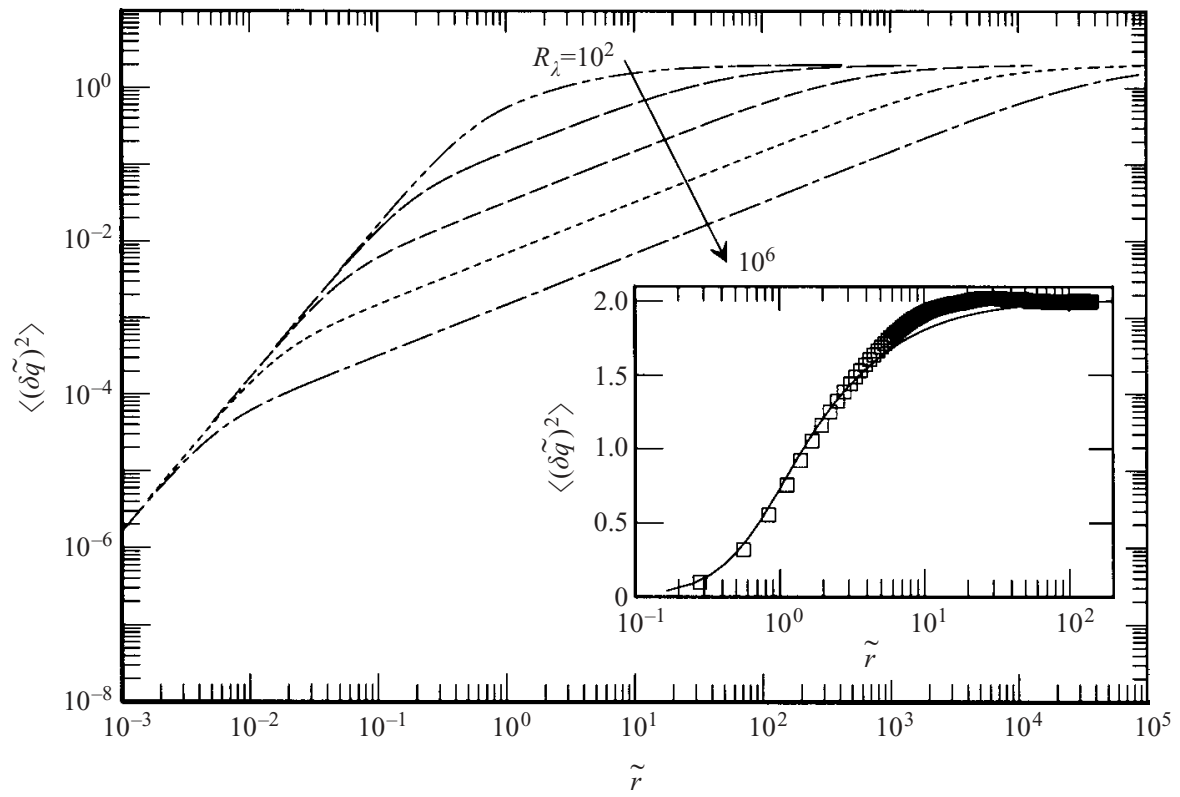

FIGURE 13. Parameterized distributions of $f_{\text {par }}\left(\equiv\left\langle(\delta \tilde{q})^{2}\right\rangle\right)$, using (6.3), over a large range of $R_{\lambda}$. - - , $R_{\lambda}=10^{6} ;---, 10^{5} ;--, 10^{4} ;-\longrightarrow, 10^{3} ;-\cdots, 10^{2}$. The inset compares $f_{\text {par }}$ at $R_{\lambda}=40(-)$ with the measured distribution of $\left\langle(\delta \tilde{q})^{2}\right\rangle$ at $x / M=50\left(R_{\lambda} \simeq 40\right)(\square)$, using the same axes as in figure $7(a)$.

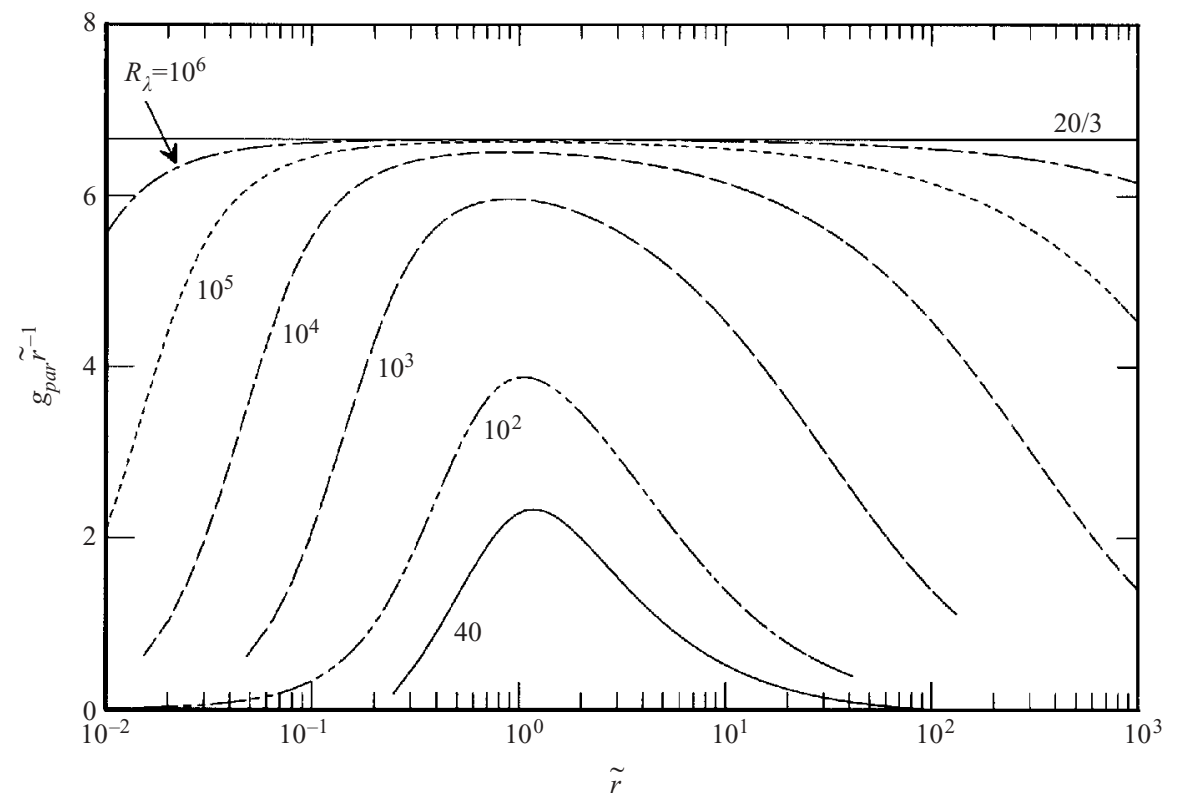

FIGURE 14. Calculated distributions of $g_{\text {par }} \tilde{r}^{-1}$, based on (2.19) and $f_{\text {par }}$. Symbols are as in figure 13 .

a plateau. If one accepts that constancy of $g \tilde{r}^{-1}$ is an appropriate indicator of the IR (the linear dependence of $g$ on $\tilde{r}$ is not affected by intermittency), the distributions in figure 14 suggest that $R_{\lambda}$ needs to be equal to about $10^{6}$ before a significant IR, over 
approximately two decades in $\tilde{r}$, is established. The location of the peak in $g \tilde{r}^{-1}$ or that of the centre of the plateau remains at $\tilde{r} \simeq 1$, independently of $R_{\lambda}$.

\section{Concluding discussion}

The analysis and arguments presented in $\S 2$ indicate that similarity of the transport equation of $\left\langle(\delta q)^{2}\right\rangle$ for decaying homogeneous isotropic turbulence is possible when the characteristic velocity scale $\left\langle q^{2}\right\rangle^{1 / 2}$ decays in power-law fashion, i.e. $\left\langle q^{2}\right\rangle \sim x^{m}$, and the characteristic length scale, which is identifiable with the Taylor microscale, grows as $x^{1 / 2}$. The turbulence Reynolds number $R_{\lambda}$ can decay with $x$ if the exponent $m$ is smaller than -1 . These results are in full accord with the results obtained by George (1992) by considering the spectral energy equation. When $m$ is -1 , a result expected to occur asymptotically $\left(R_{\lambda} \rightarrow \infty\right)$, the present similarity solution, like George's, would be consistent with the predictions of Kármán \& Howarth (1938), Dryden (1948) and Speziale \& Bernard (1992).

In realizations of decaying homogeneous isotropic turbulence, whether they are carried out in the laboratory or on a computer, $m$ is typically smaller than -1 and $R_{\lambda}$ decays with $x$ (or time), albeit slowly. Such a behaviour is consistent with our analysis. In turbulent grid flow, the assumptions of homogeneity and isotropy, which are required in the theory, are violated at large scales and satisfied approximately at small scales. Although the Taylor microscale $\lambda$ grows as $x^{1 / 2}-$ this follows from the definition of $\lambda$ and the power-law variation of $\left\langle q^{2}\right\rangle$ - the measured values of $L_{\alpha}$ and $\eta$ grow at slower and faster rates respectively than $\lambda$. The latter result is as expected since, for isotropic turbulence, $\eta / \lambda \sim R_{\lambda}^{-1 / 2}$ and $R_{\lambda}$ decays as $x^{(m+1) / 2}$. The former result very likely reflects the effect on the largest flow scales of the tunnel walls. DNS data for decaying turbulence appear to also be affected in a similar way, due to the finite size of the box, e.g. George et al. (2001) and Wang \& George (2002).

Despite the limitations of the experiment, the distributions of $\left\langle(\delta q)^{2}\right\rangle$, when normalized by $\left\langle q^{2}\right\rangle$ and $\lambda$, satisfy similarity reasonably well over a significant range of scales, thus providing support for the analysis. Kolmogorov-normalized distributions of $\left\langle(\delta q)^{2}\right\rangle$ collapse only at small $r$ for the present small value of $R_{M}$. Despite $R_{M}$ being small, this collapse is consistent, in spirit, with Kolmogorov's (1941c) first similarity hypothesis, mainly because the small scales are nearly isotropic in this flow. Kolmogorov-normalization breaks down at sufficiently large $r$ since the ratio $\left\langle q^{* 2}\right\rangle \equiv\left\langle q^{2}\right\rangle / U_{K}^{2}$ evolves linearly with $R_{\lambda}$; this local sensitivity may be regarded as one advantage of structure functions over spectra since, for the latter, it is the integral under the spectrum which is equal to $\left\langle q^{* 2}\right\rangle$. The range of $r$ for which Kolmogorov normalization applies should expand as $R_{\lambda}$ increases. If the solution $m=-1$ corresponds to very large $R_{\lambda}$ (as discussed in G92), Kolmogorov normalization would then be fully consistent with a similarity based on $\left\langle q^{2}\right\rangle$ and $\lambda$ (or $L$ ). For a given $R_{M}$, even when the latter is small, there is a range for which Kolmogorov's (1941c) similarity hypothesis is consistent with the solution of $\S 2$. This range extends as $R_{M}$ increases. A solution based on Kolmogorov's (1941b) equation, which ignores $\Gamma_{1}$ and $\Gamma_{2}$, is relevant at very large $R_{M}$.

The collapse of the $\lambda$-normalized $\left\langle(\delta q)^{2}\right\rangle$ data provides an important platform for the purpose of calculating $g \equiv-3^{1 / 2} R_{\lambda}\left\langle(\delta \tilde{u})(\delta \tilde{q})^{2}\right\rangle$, the normalized third-order structure function. Agreement between calculated and measured distributions of $g$ is satisfactory, allowing for the uncertainty in this measurement, the fact that homogeneity and isotropy are satisfied only approximately in the experiment and the possible influence of the tunnel walls. The level of agreement is an improvement over 
that previously reported between calculated and measured third-order correlation functions. It is also comparable to that found by Wang et al. (2000) between DNS and calculated distributions of $T(k)$.

The calculation of $g$, based on the present similarity solution and a parametric relation for $f$, has been extended to large values of $R_{\lambda}$. The results underline that, for decaying homogeneous isotropic turbulence, a very large value of $R_{\lambda}$, typically about $10^{6}$, is needed before a significant (two decades in $\tilde{r}$ ) inertial range, where $\tilde{g}$ is proportional to $\tilde{r}$ (Kolmogorov 1941b), may be claimed. The midpoint of the inertial range $(\tilde{r} \simeq 1)$ at $R_{\lambda}=10^{6}$ also corresponds to the maximum in $g \tilde{r}^{-1}$ at much lower $R_{\lambda}$. This behaviour appears to reflect the relevance of $\lambda$, which involves both the turbulent energy $\left\langle q^{2}\right\rangle$, representing mainly the contribution from large scales, and $\langle\epsilon\rangle$ which is a measure of the energy dissipated by the small scales as well as of the rate at which energy is transferred down the turbulence cascade.

R.A.A. acknowledges the support of the Australian Research Council. R.A.A. and R.J.S. are very grateful to W. K. George for many interesting discussions.

\section{REFERENCES}

Antonia, R. A. 2000 Reynolds number dependence of velocity and temperature increments in turbulent flows. In Turbulence, Heat and Mass Transfer 3 (ed. Y. Nagano, K. Hanjalic \& T. Tsuji), pp. 3-14. Aichi Shuppan.

Antonia, R. A., ORlandi, P. \& Zhou, T. $2002 a$ Assessment of a three-component vorticity probe in decaying turbulence. Exps. Fluids 33, 384-390.

Antonia, R. A. \& Pearson, B. R. 2000 Effect of initial conditions on the mean energy dissipation rate and the scaling exponent. Phys. Rev. E 62, 8086-8090.

Antonia, R. A., Zhou, T. \& Romano, G. P. $2002 b$ Small-scale turbulence characteristics of twodimensional bluff body wakes. J. Fluid Mech. 459, 67-92.

Antonia, R. A., Zhou, T. \& ZhU, Y. 1998 Three-component vorticity measurements in a turbulent grid flow. J. Fluid Mech. 374, 29-57.

BATCHELOR, G. K. 1948 Energy decay and self-preserving correlation functions in isotropic turbulence. Q. Appl. Maths 6, 97-116.

Batchelor, G. K. 1951 Pressure fluctuations in isotropic turbulence. Proc. Camb. Phil. Soc. 47, 359-374

Batchelor, G. K. 1953 The Theory of Homogeneous Turbulence. Cambridge University Press.

Batchelor, G. K. \& Townsend, A. A. 1947 Decay of vorticity in isotropic turbulence. Proc. R. Soc. Lond. A 191, 534-550.

Batchelor, G. K. \& Townsend, A. A. 1948 Decay of isotropic turbulence in the initial period. Proc. R. Soc. Lond. A 193, 539-558.

Comte-Bellot, G. \& Corrsin, S. 1966 The use of a contraction to improve the isotropy of grid-generated turbulence. J. Fluid Mech. 25, 657-682.

Comte-Bellot, G. \& Corrsin, S. 1971 Simple Eulerian time correlation of full- and narrow-band velocity signals in grid-generated, 'isotropic' turbulence. J. Fluid Mech. 48, 273-337.

Corrsin, S. 1963 Turbulence: experimental methods. In Handbuch der Physik (ed. S. Flügge \& C. A. Truesdell), Vol. II/2, pp. 524-589. Springer.

Danaila, L., Anselmet, F. \& Antonia, R. A. 2002 An overview of the effect of large-scale nonhomogeneities on small-scale turbulence. Phys. Fluids 14, 2475-2484.

Danaila, L., Anselmet, F., Zhou, T. \& Antonia, R. A. 1999 A generalization of Yaglom's equation which accounts for the large-scale forcing in heated grid turbulence. J. Fluid Mech. 391, 359-372.

Dhruva, B. 2000 An experimental study of high Reynolds number turbulence in the atmosphere. $\mathrm{PhD}$ Thesis, Yale University.

DrYDEN, H. L. 1943 A review of the statistical theory of turbulence. Q. Appl. Maths 1, 7-42. 
Fulachier, L. \& Antonia, R. A. 1983 Turbulent Reynolds and Péclet numbers re-defined. Intl Commun. Heat Mass Transfer 10, 435-439.

George, W. K. 1987 A theory for the decay of homogeneous isotropic turbulence. Tech. Rep. 116, State Univ. of NY at Buffalo. (A short version of this report appeared in Transport Phenomena in Turbulent Flows (ed. M. Hirata \& N. Kasagi), pp. 3-16, Hemisphere, 1988.)

George, W. K. 1992 The decay of homogeneous isotropic turbulence. Phys. Fluids A 4, 1492-1509 (referred to herein as G92).

George, W. K. 1999 Some thoughts on similarity, the POD, and finite boundaries. In Trends in Mathematics: Fundamental Problematic Issues in Turbulence (ed. A. Gyr, W. Kinzelbach \& A. Tsinober), pp. 117-128. Birkhäuser.

George, W. K., Wang, H., Wollblad, C. \& Johansson, T. G. 2001 Homogeneous turbulence and its relation to realizable flows. Proc. 14th Australasian Fluid Mechanics Conference (ed. B. B. Dally), pp. 41-48. Adelaide University.

GoldSTEIN, S. 1951 On the law of decay of homogeneous isotropic turbulence and the theories of the equilibrium and similarity spectra. Proc. Camb. Phil. Soc. 47, 554-574.

HinZe, J. O. 1975 Turbulence. McGraw-Hill.

von KÁrmán, T. \& Howarth, L. 1938 On the statistical theory of isotropic turbulence. Proc. R. Soc. Lond. A 164, 192-215.

VON KÁRMÁn, T. \& LIN, C. C. 1949 On the concept of similarity in the theory of isotropic turbulence. Rev. Mod. Phys. 21, 516-519.

Kolmogorov, A. N. 1941a On degeneration of isotropic turbulence in an incompressible viscous fluid. Dokl. Akad. Nauk. SSSR 31, 538-540.

Kolmogorov, A. N. $1941 b$ Dissipation of energy in locally isotropic turbulence. Dokl. Akad. Nauk. SSSR 32, 16-18.

Kolmogorov, A. N. 1941c The local structure of turbulence in an incompressible fluid with very large Reynolds numbers. Dokl. Akad. Nauk. SSSR 30, 301-305.

Kurien, S. \& Sreenivasan, K. R. 2000 Anisotropic scaling contributions to high-order structure functions in high Reynolds number turbulence. Phys. Rev. E 62, 2206-2212.

Lin, C. C. 1948 Note on the law of decay of isotropic turbulence. Proc. Natl Acad. Sci. 34, 540 543.

Mohamed, M. S. \& LaRue, J. C. 1990 The decay power-law in grid generated turbulence. J. Fluid Mech. 219, 195-214.

Monin, A. S. \& Yaglom, A. M. 1975 Statistical Fluid Mechanics, Vol. 2. MIT Press.

MydLARKSI, L. \& WARHAFT, Z. 1996 On the onset of high-Reynolds-number grid-generated wind tunnel turbulence. J. Fluid Mech. 320, 331-368.

Panchev, P. 1971 Random Functions and Turbulence. Pergamon.

Proudman, I. \& ReID, W. H. 1954 On the decay of a normally distributed and homogeneous turbulent velocity field. Proc. R. Soc. Lond. A 247, 163-189.

Saddoughi, S. G. \& Veeravalli, S. V. 1994 Local isotropy in turbulent boundary layers at high Reynolds number. J. Fluid Mech. 268, 333-372.

SAfFman, P. G. 1967 The large scale structure of homogeneous turbulence. J. Fluid Mech. 27, 581-593.

Speziale, C. G. \& Bernard, P. S. 1992 The energy decay in self-preserving isotropic turbulence revisited. J. Fluid Mech. 241, 645-667.

Sreenivasan, K. R. 1984 On the scaling of the energy dissipation rate. Phys. Fluids 27, 1048-1051.

Stewart, R. W. 1951 Triple velocity correlations in isotropic turbulence. Proc. Camb. Phil. Soc. 47, $146-157$.

Stewart, R. W. \& Townsend, A. A. 1951 Similarity and self-preservation in isotropic turbulence. Phil. Trans. R. Soc. A 243, 359-386.

Stolovitzky, G., Sreenivasan, K. R. \& Juneja, A. 1993 Scaling functions and scaling exponents in turbulence. Phys. Rev. E 48, R3217-R3220.

Tatsumi, T. 1980 Theory of homogeneous turbulence. Adv. Appl. Mech. 20, 39-133.

TAYLOR, G. I. 1935 Statistical theory of isotropic turbulence. Parts I-IV. Proc. R. Soc. Lond. A 151, $421-478$.

Tennekes, H. \& Lumley, J. L. 1972 A First Course in Turbulence. MIT Press. 
Wang, H. \& George, W. K. 2002 The integral scale in homogeneous isotropic turbulence. J. Fluid Mech. 459, 429-443.

Zhou, T. \& Antonia, R. A. 2000 Reynolds number dependence of the small scale structure of grid turbulence. J. Fluid Mech. 406, 81-107.

Zhou, T., Antonia, R. A., Danaila, L. \& Anselmet, F. 2000 Transport equations for the mean energy and temperature dissipation rates in grid turbulence. Exps. Fluids 28, 143-151.

Zhou, Y. \& Speziale, C. G. 1998 Advances in the fundamental aspects of turbulence : energy transfer, interacting scales, and self-preservation in isotropic decay. Appl. Mech. Rev. 51, 267-301. 